\title{
Conditional Bursting Enhances Resonant Firing in Neocortical Layer 2-3 Pyramidal Neurons
}

\author{
Matthew H. Higgs ${ }^{1,2}$ and William J. Spain ${ }^{1,2,3}$ \\ ${ }^{1}$ Neurology Section, Veterans Affairs Puget Sound Health Care System, Seattle, Washington 98108, and Departments of ${ }^{2}$ Physiology and Biophysics and \\ ${ }^{3}$ Neurology, University of Washington, Seattle, Washington 98195
}

The frequency response properties of neurons are critical for signal transmission and control of network oscillations. At subthreshold membrane potential, some neurons show resonance caused by voltage-gated channels. During action potential firing, resonance of the spike output may arise from subthreshold mechanisms and/or spike-dependent currents that cause afterhyperpolarizations (AHPs) and afterdepolarizations (ADPs). Layer 2-3 pyramidal neurons (L2-3 PNs) have a fast ADP that can trigger bursts. The present study investigated what stimuli elicit bursting in these cells and whether bursts transmit specific frequency components of the synaptic input, leading to resonance at particular frequencies. We found that two-spike bursts are triggered by step onsets, sine waves in two frequency bands, and noise. Using noise adjusted to elicit firing at $\sim 10 \mathrm{~Hz}$, we measured the gain for modulation of the time-varying firing rate as a function of stimulus frequency, finding a primary peak $(7-16 \mathrm{~Hz})$ and a high-frequency resonance $(250-450 \mathrm{~Hz})$. Gain was also measured separately for single and burst spikes. For a given spike rate, bursts provided higher gain at the primary peak and lower gain at intermediate frequencies, sharpening the high-frequency resonance. Suppression of bursting using automated current feedback weakened the primary and high-frequency resonances. The primary resonance was also influenced by the SK channel-mediated medium AHP (mAHP), because the SK blocker apamin reduced the sharpness of the primary peak. Our results suggest that resonance in L2-3 PNs depends on burst firing and the mAHP. Bursting enhances resonance in two distinct frequency bands.

Key words: burst; resonance; oscillator; frequency; cortex; pyramidal cell

\section{Introduction}

The ability of neurons to transmit transient signals and oscillations depends on their frequency response properties. Neuronal frequency tuning is often investigated at subthreshold membrane potential $\left(V_{m}\right)$, by measuring the frequency-dependent impedance magnitude, $Z(f)$, the ratio of sinusoidal $V_{m}$ fluctuation to sine wave current. A peak of $Z(f)$ shows that a neuron has subthreshold resonance, behaving as a bandpass filter. Subthreshold resonance is observed in some hippocampal and cortical pyramidal neurons (PNs) (Gutfreund et al., 1995; Hutcheon et al., 1996; Leung and Yu, 1998; Hu et al., 2002; Peters et al., 2005; Narayanan and Johnston, 2007) but not in others (Haas and White, 2002).

The frequency response for spike output may be quantified by the frequency-dependent gain, $G(f)$, the ratio of sinusoidal firing rate modulation to sine wave current. $G(f)$ is an appropriate measure when each frequency component of the input is small relative to the overall fluctuation, causing sinusoidal variation of firing probability rather than phase-locked spikes (Yu and Lewis,

Received Aug. 6, 2008; revised Dec. 10, 2008; accepted Dec. 10, 2008.

This work was supported by a Veterans Affairs Merit Review and an Marine Biological Laboratory Fellowship (W.J.S.). We thank Sean Slee, Brian Lundstrom, Fred Rieke, Adrienne Fairhall, Mark Binder, Dan Johnston, and Eric Shea-Brown for helpful discussions and Susan Usher for excellent technical assistance.

Correspondence should be addressed to William J. Spain, Veterans Affairs Puget Sound Health Care System, Neurology (Mail Stop 127), 1660 South Columbian Way, Seattle, WA 98018. E-mail: spain@u.washington.edu. D0I:10.1523/JNEUROSCI.3728-08.2009

Copyright $\odot 2009$ Society for Neuroscience $\quad$ 0270-6474/09/291285-15\$15.00/0
1989). $G(f)$ behaves differently from $Z(f)$ because of the nonlinearity of spike generation and the feedback mechanisms activated by spikes. In cortical PNs, $G(f)$ remains high above $100 \mathrm{~Hz}$, at which subthreshold $V_{m}$ responses are attenuated because of the membrane time constant (Carandini et al., 1996; Köndgen et al., 2008). Evidently, small, high-frequency $V_{m}$ fluctuations efficiently modulate firing probability. At very high frequency, the fall of $G(f)$ depends on the dynamics of spike generation (Fourcaud-Trocmé et al., 2003).

With some neuronal properties and stimulus parameters, $G(f)$ shows a peak, or resonance. Modeling results show that resonance can arise from subthreshold voltage-dependent currents (Brunel et al., 2003; Richardson et al., 2003) and/or spike afterhyperpolarizations (AHPs) (Fuhrmann et al., 2002). Theory and models suggest that an AHP of duration well matched to the mean interspike interval (ISI) regularizes the spike train, causing resonance at the mean firing rate $\left(r_{0}\right)$ and multiples thereof. Noise reduces this resonance by disrupting the regularity of firing (Brunel et al., 2001) but drives resonance governed by voltagedependent currents (Brunel et al., 2003; Richardson et al., 2003).

The present study investigated the hypothesis that the fast afterdepolarization (fADP) and bursting seen in many cortical PNs strengthen firing resonance. PNs show a spectrum of bursting tendencies. Repetitive bursting in response to constant current is observed in "intrinsically bursting" PNs in layer 5 (Connors et al., 1982) and "chattering cells" in layer 2-3 (L2-3) (Gray and McCormick, 1996) and other layers (Steriade et al., 1998a). 
Weaker bursting is seen in "regular-spiking" (RS) PNs that burst to sine waves of certain frequencies, noise (Schindler et al., 2006), or natural synaptic input (Chen and Fetz, 2005; de Kock and Sakmann, 2008). In the present study, we observed that RS PNs in L2-3 of rat motor and somatosensory cortex burst when subjected to fluctuating stimuli. Bursts provide high gain at specific frequencies and strengthen resonance in two distinct frequency bands. Our results suggest that bursting in L2-3 PNs may promote transmission of both slow and fast oscillations within cortical circuits.

\section{Materials and Methods}

Preparation of cortical slices. Six- to 12-week-old male Sprague Dawley rats were deeply anesthetized with isoflurane $(4 \%)$ in oxygen and quickly decapitated. The brain was removed rapidly and chilled for $\sim 1 \mathrm{~min}$ in ice-cold cutting solution containing the following (in mM): 220 sucrose, $3 \mathrm{KCl}, 1 \mathrm{CaCl}_{2}, 5 \mathrm{MgCl}_{2}, 26 \mathrm{NaHCO}_{3}, 1.25 \mathrm{NaH}_{2} \mathrm{PO}_{4}$, and 10 D-glucose [bubbled with $95 \% \mathrm{O}_{2}, 5 \% \mathrm{CO}_{2}$ (carbogen)]. A block of the brain containing the motor and somatosensory cortex was attached to the stage of a vibrating tissue slicer (TPI) with cyanoacrylate glue, immersed in icecold cutting solution, and bisected sagittally. Five $300 \mu \mathrm{m}$ coronal slices were obtained, from $\sim 0$ to $-1.5 \mathrm{~mm}$ relative to bregma (Paxinos and Watson, 1986). Slices were transferred to a holding chamber filled with artificial CSF (ACSF) containing the following (in mM): $125 \mathrm{NaCl}, 3 \mathrm{KCl}$, $2 \mathrm{CaCl}_{2}, 2 \mathrm{MgCl}_{2}, 26 \mathrm{NaHCO}_{3}, 1.25 \mathrm{NaH}_{2} \mathrm{PO}_{4}$, and 20 D-glucose, bubbled with carbogen. The holding chamber was kept at $34^{\circ} \mathrm{C}$ for $1 \mathrm{~h}$ and then allowed to cool to room temperature.

Recording. Intracellular recordings were obtained from RS neurons in layer 2-3 of the dorsal neocortex using sharp electrodes (40-100 $\mathrm{M} \Omega$ ) filled with $3 \mathrm{M} \mathrm{KCl}$. Most of the cells were located in the forelimb and hindlimb motor areas, based on the rat brain atlas of Paxinos and Watson (1986). In some experiments, the electrode solution contained $1 \%$ biocytin for subsequent visualization of recorded cells. For experiments, a slice was transferred to a submerged recording chamber and perfused at $\sim 2 \mathrm{ml} / \mathrm{min}$ with ACSF $\left(34^{\circ} \mathrm{C}\right)$ containing DNQX $(20 \mu \mathrm{M}),( \pm) 3-(2-$ carboxypiperazin-4-yl)-propyl-1-phosphonic acid (5 $\mu \mathrm{M})$, and picrotoxin $(100 \mu \mathrm{M})$ to block AMPA/kainate, NMDA, and $\mathrm{GABA}_{\mathrm{A}}$ receptors, respectively. Current injection and voltage recording were performed using an Axoclamp-2A amplifier (Molecular Devices) in continuous bridge mode. Data were filtered at $10 \mathrm{kHz}$ using the single-pole low-pass filter of the Axoclamp-2A and sampled at $20 \mathrm{kHz}$ using an ITC-16 or ITC-18 data acquisition board (InstruTECH Corporation) connected to a Macintosh computer (Apple Computers). Current injection and data acquisition were controlled by custom macros in Igor Pro (WaveMetrics).

Confirmation of cell layer and type. In a subset of experiments, biocytin was included in the electrode solution to confirm the cortical location and cell type. After recording from one cell, the slice was fixed overnight in $4 \%$ paraformaldehyde and the cell was visualized using the Vectastain ABC Elite kit (Vector Laboratories), followed by development with diaminobenzidine and $\mathrm{H}_{2} \mathrm{O}_{2}$ (Sigma Fast kit).

Current stimuli. The basic subthreshold membrane properties and repetitive firing of each neuron were characterized by injecting a series of increasing $1 \mathrm{~s}$ current steps at a $20 \mathrm{~s}$ interval. To investigate frequency response properties, three types of stimuli were applied: (1) sinusoidal chirp current of varying frequency, (2) exponential-filtered noise, and (3) " $1 / f$ noise," the power of which varies inversely with frequency. The total stimulus current was given by the sum of direct current $\left(I_{\mathrm{DC}}\right)$ and chirp or noise current, $I_{\text {chirp }}(t)$ or $I_{\text {noise }}(t)$. In most experiments, $I_{\mathrm{DC}}$ was set slightly below the current threshold of the cell (rheobase).

Chirps were described by the following:

$$
\begin{aligned}
I_{\text {chirp }}(t)= & A \sin [2 \pi n(t)], \\
n(t)= & { }^{t} f(t) d t,
\end{aligned}
$$

where $A$ is the magnitude, $n(t)$ is the wave number, and $f(t)$ is the timevarying frequency. In most studies, $f(t)=10^{t / 20 \mathrm{~s}}$ for $0<t<60 \mathrm{~s}$, giving an exponential increase from 1 to $1000 \mathrm{~Hz}$. For measurements of subthreshold impedance (described below), $f(t)=(t / 60 \mathrm{~s})(30 \mathrm{~Hz})$ for $0<$ $t<60 \mathrm{~s}$, giving a linear increase from 0 to $30 \mathrm{~Hz}$.

Noise stimuli were created using the random number generator in Igor Pro. Exponential-filtered noise was generated by convolving a series of independent, Gaussian-distributed noise samples (white noise) with a decaying exponential, $\exp \left(-t / \tau_{\text {filter }}\right)$, where $\tau_{\text {filter }}=1$ or $5 \mathrm{~ms}$ as indicated. The exponential-filtered noise is equivalent to noise created by an Ornstein-Uhlenbeck process.

$1 / f$ noise was created in the frequency domain. Random phases, $-\pi<$ $\phi(f)<\pi$, were chosen from a uniform distribution, and the amplitude of each component was scaled by the following:

$$
A(f)=\left\{\begin{array}{cc}
f^{-1 / 2} & 0.05 \mathrm{~Hz} \leq f \leq 10000 \mathrm{~Hz} \\
0.05^{-1 / 2} & f<0.05 \mathrm{~Hz}
\end{array}\right\} .
$$

The real component, $R(f)$, and imaginary component, $I(f)$, at each frequency were calculated as follows:

$$
\begin{gathered}
R(f)=A(f) \cos \phi(f), \\
I(f)=A(f) \sin \phi(f) .
\end{gathered}
$$

The $1 / f$ noise was then inverse Fourier transformed to obtain the timedomain stimulus and scaled as indicated.

Automated firing rate targeting. Because theoretical and modeling results show that firing resonance depends on the mean firing rate $\left(r_{0}\right)$ (Brunel et al., 2001), some experiments were performed using a computer feedback algorithm to adjust $I_{\mathrm{DC}}$ slowly to achieve and maintain a target mean rate $\left(r_{\text {target }}\right)$ while stimulating a neuron with noisy current. Adjustment of $I_{\mathrm{DC}}$ was performed at $\sim 10 \mathrm{~s}$ intervals (varying slightly as data read/write operations were performed in blocks). Each adjustment was calculated based on the firing rate "error" and a feedback gain, $g_{\text {feed }}$ back $=-5 \mathrm{pA} / \mathrm{Hz}$ :

$$
\Delta I_{\mathrm{DC}}=g_{\text {feedback }}\left(r_{0}-r_{\text {target }}\right) .
$$

Burst termination by automated current feedback. To manipulate the fADP and consequent burst firing, a real-time feedback method was used to inject a pulse of hyperpolarizing current immediately after each spike. The circuit used comprised an analog spike detector, a pulse generator, a parallel resistor-capacitor (RC) circuit, and a summing amplifier. On the down stroke of each detected spike, at $V_{m}=-10 \mathrm{mV}$, the spike detector output triggers the pulse generator, which sends a charging pulse through an input resistor to the RC circuit. The summing amplifier adds the voltage of the $\mathrm{RC}$ circuit to the current command sent from the ITC-16 interface to the Axoclamp 2-A amplifier. The output of the pulse generator was adjusted such that the circuit generated a peak current of $-1 \mathrm{nA}$ after each spike, rising in $0.2 \mathrm{~ms}$ (the length of the charging pulse) and decaying exponentially with a $5 \mathrm{~ms}$ time constant (governed by the $\mathrm{RC}$ circuit). Hyperpolarizing current pulses of this amplitude and duration effectively opposed the fADP and essentially eliminated burst firing (see Results).

General methods of analysis. Data were analyzed using custom macros in Igor Pro. The membrane resistance $\left(R_{m}\right)$ of each cell was measured from the baseline membrane potential $\left(V_{\text {rest }}\right)$ and the mean steady-state potential $\left(V_{\mathrm{ss}}\right) 900-1000 \mathrm{~ms}$ after a step onset:

$$
R_{m}=\left(V_{\text {ss }}-V_{\text {rest }}\right) / I \text {. }
$$

The membrane time constant $\left(\tau_{m}\right)$ was estimated by fitting a single exponential from 1 to $50 \mathrm{~ms}$ after step onset:

$$
V_{m}(t)=V_{\text {rest }}+A\left[1-\exp \left(-t / \tau_{m}\right)\right] \text {. }
$$

The $R_{m}$ and $\tau_{m}$ data reported were obtained for the largest depolarizing step that did not elicit any spikes. For analyses of action potential firing, spikes were detected at a threshold of $-10 \mathrm{mV}$. Each spike time was taken at the crossing of the detection threshold, and the search for threshold crossings resumed $2 \mathrm{~ms}$ later. 
Measurement of frequency-dependent gain, $G(f)$, and mean phase shift, $\phi(f)$. The linear component of the spike response to a noise stimulus was quantified by correlation analysis (Lee and Schetzen, 1965; Guttman et al., 1974; Bryant and Segundo, 1976). Because the neuron is a nonlinear system, the values of $G(f)$ and $\phi(f)$ are specific to the stimulus applied. The time-varying firing rate, $r(t)$, was obtained by digitizing the spike train with a bin width $\Delta t$ equal to the sampling interval $(0.05 \mathrm{~ms})$ :

$$
r(t)=\left\{\begin{array}{cc}
1 / \Delta t & \text { where spike detected } \\
0 & \text { where no spike detected }
\end{array}\right\} .
$$

The stimulus-response correlation $\left(c_{\mathrm{sr}}\right)$ and the stimulus autocorrelation $\left(c_{\mathrm{ss}}\right)$ were calculated as follows:

$$
\begin{aligned}
& c_{\mathrm{sr}}(\tau)=\langle s(t) r(t+\tau)\rangle, \\
& c_{\mathrm{ss}}(\tau)=\langle s(t) s(t+\tau)\rangle,
\end{aligned}
$$

where $\tau$ is the time difference, and the stimulus, $s(t)$, is $I_{\text {noise }}(t)$. After windowing of $c_{\mathrm{sr}}(\tau)$ and $c_{\mathrm{ss}}(\tau)$ (see below), the complex Fourier components $C_{\mathrm{sr}}(f)$ and $C_{\mathrm{ss}}(f)$ were obtained, and the frequency-dependent gain and the average phase shift were calculated:

$$
\begin{gathered}
G(f)=\frac{\left|C_{s r}(f)\right|}{\left|C_{s s}(f)\right|} \\
\phi(f)=\operatorname{atan} \frac{\left\{\operatorname{Im}\left[C_{s r}(f)\right]\right\}}{\left\{\operatorname{Re}\left[C_{s r}(f)\right]\right\}},
\end{gathered}
$$

where Re and Im refer to the real and imaginary parts of each Fourier component. Positive values of $\phi(f)$ indicate that the response lags behind the stimulus.

Windows for gain and phase analysis. To maximize the range of frequencies over which $G(f)$ and $\phi(f)$ could be estimated, a careful choice of analysis windows for $c_{\mathrm{sr}}(\tau)$ and $c_{\mathrm{ss}}(\tau)$ was required. For a given $f$, the window width must be $\geq 1 / f$. However, with an excessively wide window, $C_{\mathrm{sr}}(f)$ becomes dominated by noise. The solution used was to calculate the Fourier components $C_{\mathrm{sr}}(f)$ and $C_{\mathrm{ss}}(f)$ at a set of discrete frequencies, using a Gaussian window $w(\tau)$ with an SD of $\sigma=1 / f$ :

$$
\begin{gathered}
w(\tau)=\exp \frac{\left(-\tau^{2}\right)}{\left(2 \sigma^{2}\right)}=\exp \frac{\left(-f \tau^{2} \mid\right.}{2)} \\
C_{\mathrm{sr}}(f)=\int c_{\mathrm{sr}}(\tau) w(\tau) e^{-i 2 \pi f \tau} d \tau, \\
C_{\mathrm{ss}}(f)=\int c_{\mathrm{ss}}(\tau) w(\tau) e^{-i 2 \pi f \tau} d \tau .
\end{gathered}
$$

This frequency-dependent Gaussian windowing reduces the noise in each frequency component at the expense of very precise frequency resolution. The wavelet given by $w(\tau) e^{-i 2 \pi f} \tau=\exp \left(-f^{2} \tau^{2} / 2\right) e^{-i 2 \pi f} \tau$ in the time domain corresponds to a Gaussian bandpass filter in the frequency domain, with an SD of $\sigma_{f}=f /(2 \pi) \approx 0.16 f$. We measured $C_{\text {sr }}(f)$ and $C_{\text {ss }}(f)$ at frequency increments of $0.1 \log _{10}$. Wider analysis windows and finer frequency sampling did not substantially affect the measured $G(f)$ and $\phi(f)$ of L2-3 PNs, but wider windows increased the measurement noise.

Correction of $\phi(f)$ for a fixed time delay. At high frequencies, $\phi(f)$ increased approximately in proportion to $f$, suggesting that there was a fixed time delay between the spike-triggering current fluctuations and the time when each spike was detected. The same observation was reported by Köndgen et al. (2008) in their study of layer five PNs. The delay $\left(\tau_{\text {delay }}\right)$ was estimated as the peak location of $c_{\text {sr }}(\tau)$. The rising phase of $c_{\mathrm{sr}}(\tau)$ before the peak closely resembled the rising phase of $c_{\mathrm{ss}}(\tau)$, suggesting that current arriving within the delay period did not influence spike generation. The corrected phase delay was calculated as follows:

$$
\phi_{\text {corrected }}(f)=\phi(f)-360^{\circ} \times f \tau_{\text {delay }} .
$$

Measurement of the resonance peaks of $G(f)$. The $G(f)$ curves of most L2-3 PNs showed two peaks. To measure each peak, we first fitted the peak with a five-term polynomial, typically fitting over a frequency range of \pm
$0.5 \log _{10}$ from the point of highest gain. The peak frequency $\left(f_{\text {peak }}\right)$ was then taken as the peak of the fitted curve. A measure of resonance strength, or sharpness of the peak, was calculated as follows:

$$
S_{\text {res }}=\frac{G\left(f_{\text {peak }}\right)}{0.5\left[G\left(0.5 f_{\text {peak }}\right)+G\left(2 f_{\text {peak }}\right)\right]}-1
$$

Measurement of frequency-dependent membrane impedance. In several neurons, the subthreshold impedance magnitude, $Z(f)$, was measured using a chirp current stimulus increasing linearly from 0 to $30 \mathrm{~Hz}$ in $60 \mathrm{~s}$. The chirp current had a magnitude of $25-50 \mathrm{pA}$ and was added to the highest level of $I_{\mathrm{DC}}$ at which the stimulus could be applied without triggering any spikes. The calculation of $Z(f)$ was identical to that of $G(f)$ described above, except that the stimulus, $s(t)$, was the chirp current, the response, $r(t)$, was $V_{m}(t)$, and the range of frequencies analyzed was 1-29 Hz.

Modeling. To investigate the effects of the fADP and medium AHP $(\mathrm{mAHP})$ in a minimal model, we used a single-compartment, leaky integrate-and-fire (LIF) neuron with a fast ADP and/or a medium AHP. The model parameters were as follows:
Membrane capacitance:
Leak conductance:
$C_{m}=500 \mathrm{pF}$
ADP conductance increment:
AHP conductance increment:
ADP decay time constant:
$G_{\text {leak }}=20 \mathrm{nS}$
$\Delta G_{A D P}=\{0,20\} \mathrm{nS} /$ spike
$\Delta G_{\mathrm{AHP}}=\{0,5\} \mathrm{nS} /$ spike
$\tau_{\mathrm{ADP}}=1 \mathrm{~ms}$
AHP decay time constant:
$\tau_{\mathrm{AHP}}=50 \mathrm{~ms}$
Leak reversal potential:
ADP reversal potential:
AHP reversal potential:
Spike threshold:
Post-spike reset potential:
Absolute refractory period:
$E_{\text {leak }}=-80 \mathrm{mV}$
$E_{\mathrm{ADP}}=E_{\mathrm{Na}}=+70 \mathrm{mV}$
$E_{\mathrm{AHP}}=E_{K}=-100 \mathrm{mV}$
$V_{\text {threshold }}=-55 \mathrm{mV}$
$V_{\text {reset }}=-60 \mathrm{mV}$
$\Delta t_{\text {refractory }}=2 \mathrm{~ms}$.

While $V_{m}<V_{\text {threshold }}$, the model behaved according to the following:

$$
\begin{gathered}
d V_{m} / d_{t}=\left(I_{\text {stimulus }}-I_{m}\right) / C_{m}, \\
\left.E_{\text {leak }}\right)+G_{\mathrm{ADP}}\left(V_{m}-E_{\mathrm{ADP}}\right)+G_{\mathrm{A}} \\
d G_{\mathrm{ADP}} / d_{t}=-G_{\mathrm{ADP}} / \tau_{\mathrm{ADP}}, \\
d G_{\mathrm{AHP}} / d_{t}=-G_{\mathrm{AHP}} / \tau_{\mathrm{AHP}} .
\end{gathered}
$$$$
I_{m}=G_{\text {leak }}\left(V_{m}-E_{\text {leak }}\right)+G_{\mathrm{ADP}}\left(V_{m}-E_{\mathrm{ADP}}\right)+G_{\mathrm{AHP}}\left(V_{m}-E_{\mathrm{AHP}}\right),
$$

After reaching $V_{\text {threshold }}, V_{m}$ was held at $V_{\text {reset }}$ for the absolute refractory period, during which $G_{\mathrm{ADP}}$ and $G_{\mathrm{AHP}}$ accumulated from previous spikes continued to decay. At the end of the refractory period, $G_{\mathrm{ADP}}$ and $G_{\mathrm{AHP}}$ were incremented by $\Delta G_{\mathrm{ADP}}$ and $\Delta G_{\mathrm{AHP}}$, respectively, and $V_{m}$ was released. Simulations were performed using Igor Pro, by first-order Euler integration with a time increment of $0.05 \mathrm{~ms}$. The simulated time was $3000 \mathrm{~s}$ for each set of stimulus parameters.

Data and statistics. Data reported in the text are mean $\pm \mathrm{SD}$, except as indicated. Data shown in figures are mean \pm SEM. Statistical significance was evaluated using two-tailed Student's $t$ tests unless otherwise stated. Differences were considered significant when $p<0.05$.

Materials. All chemicals were obtained from Sigma, except as stated otherwise.

\section{Results}

The primary data reported were obtained by recording intracellularly from 36 neurons in layer $2 / 3$ of rat neocortical slices using sharp electrodes filled with $3 \mathrm{M} \mathrm{KCl}$. The sharp electrode method was chosen in preference to the whole-cell patch technique because the firing patterns investigated are altered by washout during whole-cell recording. The resting $V_{m}$ of these neurons was $-82 \pm 5 \mathrm{mV}$, the input resistance for depolarizing steps was $45 \pm$ $13 \mathrm{M} \Omega$, and the membrane time constant was $17 \pm 3 \mathrm{~ms}$. All of the cells fired overshooting spikes and were RS based on responses to current steps. Twelve cells were filled with biocytin 
and subsequently visualized, and all of these were found to have pyramidal morphology with the soma in L2-3.

\section{L2-3 PNs have a fast ADP and fire two- spike bursts after rapid increases in input}

The post-spike $V_{m}$ trajectories of L2-3 PNs show a small, fast AHP, followed by a prominent $\mathrm{ADDP}$, which is terminated by a scooped descent into the mAHP (Fig. $1 A$ ). At the onset of a sufficiently large current step, most L2-3 PNs generate a distinct spike doublet (Fig. $1 B$ ). In the following sections, we adopt a minimal definition of bursting and refer to such doublets and any longer clusters of spikes separated by short $(<10 \mathrm{~ms})$ ISIs as bursts. We seldom observed bursts of more than two spikes in L2-3 PNs unless the injected current was very large. An fADP is observed after the second burst spike but does not usually trigger a third spike because of summation of the mAHP current and an increase in spike threshold. One may observe that the mAHP after the initial burst is deeper than that after a single initial spike (Fig. 1C). In most L2-3 PNs (12 of 16 tested with small current increments of $20 \mathrm{pA}$ ), the transition from a single initial spike to an initial burst occurred abruptly at a sharp current threshold (Fig. 1D). On average, the threshold was $690 \pm 226 \mathrm{pA}$, or $1.47 \pm$ 0.25 times the rheobase. The intraburst ISI was $4.3 \pm 0.9 \mathrm{~ms}$ for a step just above the threshold and showed relatively little change with increasing current. The burst ISI closely corresponded to the time from the onset of the first spike to the peak of the fADP, as measured during current steps just above rheobase in the 12 neurons found to have a sharp burst threshold (Fig. $1 E)$, and a correlation was seen between the fADP time and the burst ISI across different neurons (solid regression line, $r=$ $0.77)$.

\section{L2-3 PNs burst in response to noise stimuli}

To investigate whether bursting in L2-3 $\mathrm{PNs}$ is restricted to the stimulus onset or can occur in an ongoing manner with dynamic input, we applied stimuli comprising current noise ( $\left.I_{\text {noise }}\right)$ added to direct current $\left(I_{\mathrm{DC}}\right)$. A noise stimulus simulates a barrage of correlated EPSCs, eliciting $V_{m}$ fluctuations similar to those observed in neocortical PNs during active states in vivo (Destexhe and Paré, 1999), although simple current noise does not simulate the increased conductance associated with synaptic input that arrives near the soma. In the example shown in Figure 2 , the noise was filtered with an exponential function $\left(\tau_{\text {filter }}=5\right.$ ms, simulating a typical synaptic time constant). To measure the firing pattern of each cell at a similar mean firing rate $\left(r_{0}\right)$, we began with $I_{\mathrm{DC}}$ slightly below the rheobase and $\sigma_{I} \approx 0.5 I_{\mathrm{DC}}$, and

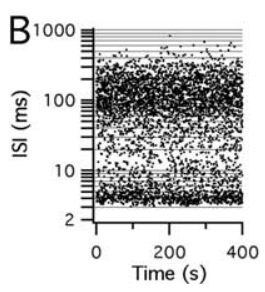

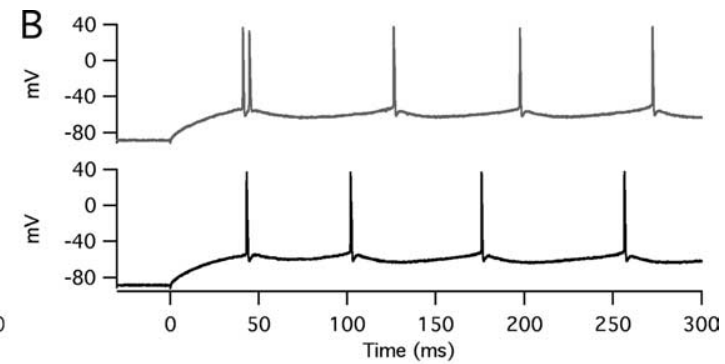
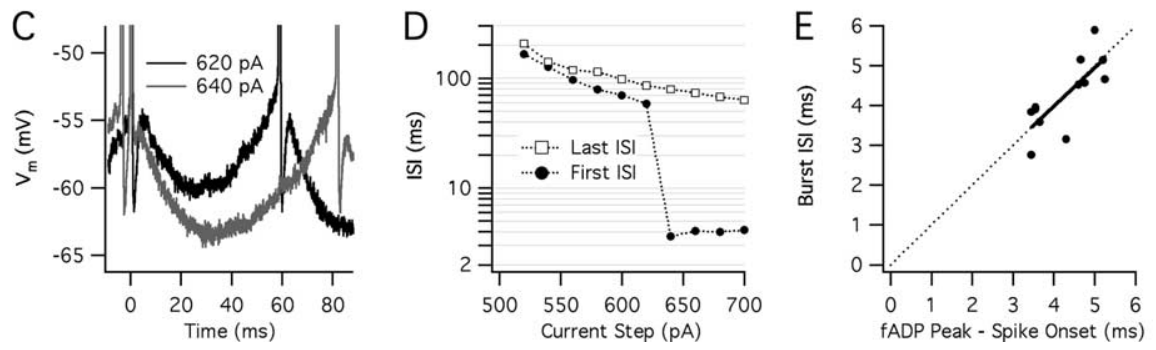

Figure 1. Spike afterpotentials and initial doublet firing. $A$, Average $V_{m}$ trajectory of a typical L2-3 PN after each spike during a current step slightly above rheobase, showing the fAHP, fADP, and mAHP. $B, A$ sharp threshold for initial doublet firing was encountered between steps of $620 \mathrm{pA}$ (black, bottom trace) and $640 \mathrm{pA}$ (gray, top trace). C, The initial doublet elicited by the 640 pA step was followed by a larger mAHP and a longer ISI than the single initial spike caused by the $620 \mathrm{pA}$ step. $\boldsymbol{D}$, First and last ISIs for a series of $1 \mathrm{~s}$ current steps. The first ISI showed a sharp change between 620 and $640 \mathrm{pA}$, whereas the last ISI did not. E, ISI for the doublet elicited by a current step just above the doublet threshold in 12 L2-3 PNs, plotted against the time from the onset of the first spike to the peak of the fADP during a current step just above rheobase. The dotted line is the line of identity. The solid line is the linear regression for the data points.
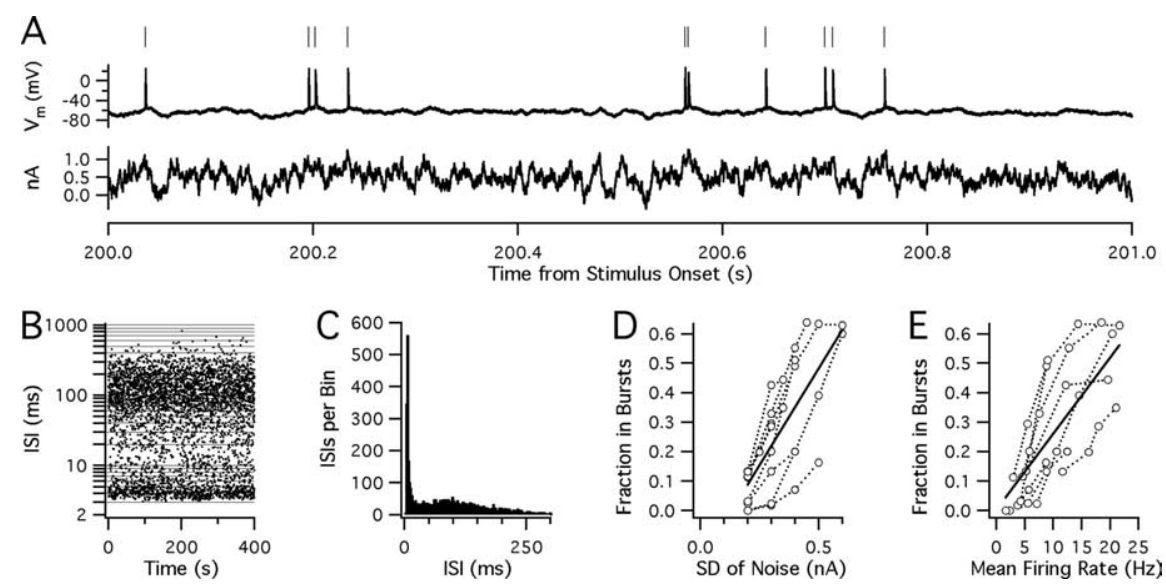

Figure 2. L2-3 PNs fire a mixture of single spikes and two-spike bursts during noise stimuli. $A$, Stimulus current (bottom), $V_{m}$ response (middle), and spike raster (top) for a cell tested with $5 \mathrm{~ms}$ exponential-filtered noise $\left(\sigma_{l}=0.25 \mathrm{nA}, I_{\mathrm{DC}}=0.47 \mathrm{nA}\right) . \boldsymbol{B}_{t}$ ISIs, plotted on a log axis, versus time during the $400 \mathrm{~s}$ stimulus. A band of short ISIs is concentrated between 3 and $6 \mathrm{~ms}$, and a broad band of long intervals is centered between 100 and $200 \mathrm{~ms}$. C, ISI histogram (2 ms bins), showing peaks of short and long intervals. $\boldsymbol{D}$, Data from eight cells subjected to $1 \mathrm{~ms}$ exponential-filtered noise, showing the fraction of spikes in bursts of two or more spikes as a function of $\sigma_{l}$. Data points from the same cell are connected by dotted lines. The solid line is the linear regression for the entire dataset. $\boldsymbol{E}$, The same data plotted as a function of the mean firing rate.

then adjusted $I_{\mathrm{DC}}$ or $\sigma_{I}$ slightly to obtain $r_{O} \approx 10 \mathrm{~Hz}$. After adjustment, in 13 cells, $r_{O}=9.95 \pm 0.49 \mathrm{~Hz}, \sigma_{I}=0.26 \pm 0.08 \mathrm{nA}$, and $I_{\mathrm{DC}}=0.46 \pm 0.20 \mathrm{nA}$. This level of stimulation was tolerated well by most L2-3 PNs; much stronger stimuli of long duration often caused a runaway increase in firing rate and long-lasting depolarization.

Figure $2 \mathrm{~A}$ shows a portion of the current stimulus (bottom), the $V_{m}$ response (middle), and the raster of detected spikes (top). The spike train was a mixture of single spikes and two-spike bursts. To examine the ISI distribution, each interval was plotted on a log scale, with the time of the second spike on the horizontal 
A

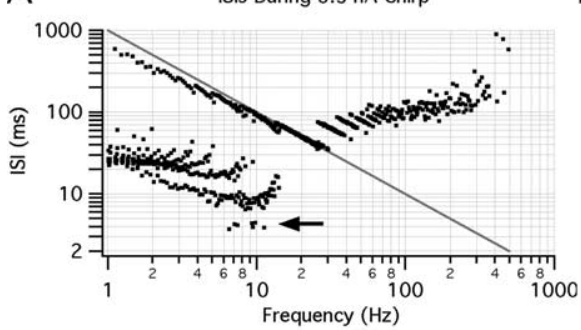

B

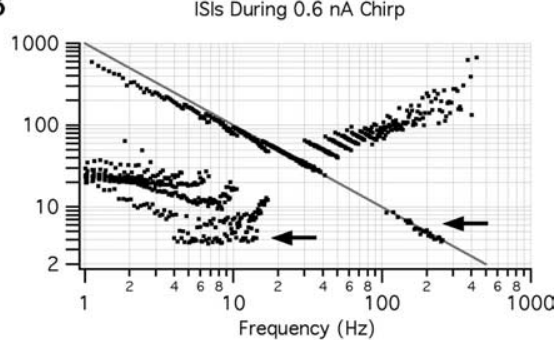

C

$0.6 \mathrm{nA}$ Chirp at $10 \mathrm{~Hz}$

D

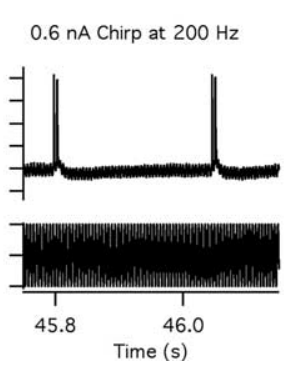

E
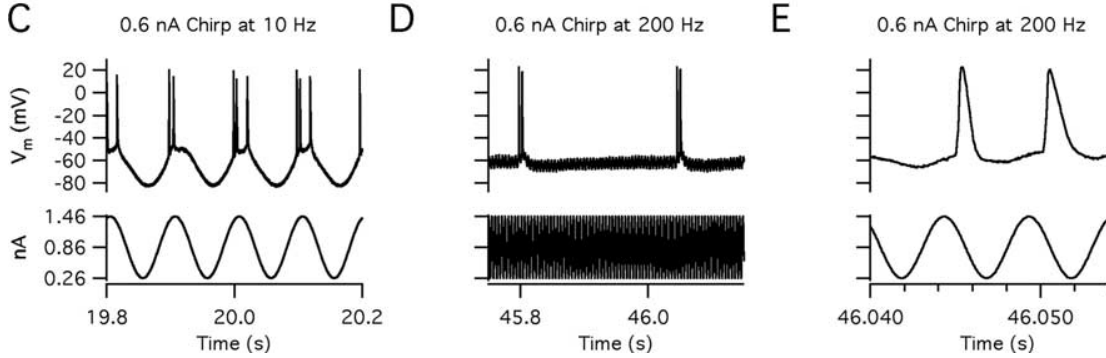

Figure 3. Two-spike bursts can be elicited by low-or high-frequency stimulus oscillations. $A$, ISIs in response to a chirp current with a magnitude of $0.5 \mathrm{nA}$, plotted against the stimulus frequency, which increased exponentially from 1 to $1000 \mathrm{~Hz}$ in $60 \mathrm{~s}$. At this stimulus magnitude, two-spike bursts (ISI $\sim 4 \mathrm{~ms}$, arrow) just began to appear at $\sim 6-12 \mathrm{~Hz}$. B, ISIs elicited by a chirp with a magnitude of $0.6 \mathrm{nA}$. A larger number of bursts were produced from $\sim 4$ to $15 \mathrm{~Hz}$, and an additional band appeared from $\sim 100$ to $250 \mathrm{~Hz}$. The ISIs of bursts elicited at low frequencies were relatively constant, whereas those obtained at high frequencies approximately matched the stimulus period (gray line). C, Response to the $0.6 \mathrm{nA}$ chirp at $10 \mathrm{~Hz}$. A two-spike burst occurred on each stimulus crest. In some cycles, this was followed by a third spike after a longer ISI. D, Response at $200 \mathrm{~Hz}$. Two-spike bursts were separated by long ISIs spanning many stimulus periods. $\boldsymbol{E}$, Expanded view of a two-spike burst at $200 \mathrm{~Hz}$. The spikes aligned with successive stimulus cycles, showing a lag relative to the peak current.

axis (Fig. 2B). The plot shows a stable firing pattern for $400 \mathrm{~s}$ of continuous stimulation. Many ISIs were clustered between 3 and $6 \mathrm{~ms}$, similar to burst intervals seen at the onset of current steps. A second, broad band of ISIs was centered at 100-200 ms. The corresponding ISI histogram (Fig. 2C) shows a large peak at 4-6 $\mathrm{ms}$, followed by a slight dip and a broad peak of long intervals. Qualitatively similar results were found in most L2-3 PNs. In the 13 L2 -3 cells tested, $26 \pm 18 \%$ of the spikes occurred in bursts, defined by a preceding and/or after ISI $<10 \mathrm{~ms}$. On average, $0.3 \%$ of all spikes were found in the middle of a burst, both preceded and followed by an ISI $<10 \mathrm{~ms}$. Thus, $\sim 1 \%$ of spikes occurred in bursts of at least three spikes.

When $\sigma_{I}$ was varied, keeping $I_{\mathrm{DC}}$ constant, the fraction of spikes in bursts increased as a function of $\sigma_{I}$ (Fig. $2 D$ ) and the resulting $r_{O}$ (Fig. $2 E$ ). The effect of changing $I_{\mathrm{DC}}$ was more variable. In general, increasing $I_{\mathrm{DC}}$ at levels below the rheobase caused a greater fraction of spikes to occur in bursts, whereas larger increases often suppressed bursting.

\section{Bursts can be elicited by low- or high-frequency stimulus oscillations}

Bursting may contribute to the firing resonances of a neuron in two ways. First, entire bursts may occur on the crests or rising phases of input oscillations with a period much longer than the intraburst intervals. Second, bursts may align with fast stimulus oscillations that match the intraburst intervals. As an initial method to investigate what oscillation frequencies can elicit bursts, we injected sinusoidal currents of varying frequency (chirps), increasing exponentially from 1 to $1000 \mathrm{~Hz}$ in $1 \mathrm{~min}$ (1 $\log / 20 \mathrm{~s}$ ). The chirp was added to $I_{\mathrm{DC}}$ set just below the rheobase, and its magnitude was increased in successive trials.

In most cells, two-spike bursts with short ISIs first appeared at $\sim 10 \mathrm{~Hz}$ (Fig. $3 A, C$ ). At lower frequencies, each stimulus oscilla- tion elicited multiple spikes at longer ISIs. At higher frequencies, each cycle triggered a maximum of one spike. When the magnitude of the chirp was increased further, oscillations of $100-250 \mathrm{~Hz}$ also caused bursting (Fig. $3 B, D, E$ ). Bursts elicited by slow sinusoids had relatively constant ISIs, whereas those triggered by fast sinusoids showed a range of ISIs approximately matching the stimulus period. Similar results were observed in five of seven cells. In the other two cells, bursts were not obtained at chirp magnitudes up to $0.6 \mathrm{nA}$. These results indicate that, in most L2-3 PNs, bursts can be elicited by low- or highfrequency stimulus oscillations and might therefore promote transmission of two frequency bands within a complex input. Similar responses to chirps were reported previously for L5 PNs (Schindler et al., 2006). However, these authors focused on the bursting elicited by high stimulus frequencies, because the firing patterns in response to slower sinusoids did not conform to their definition of bursting.

\section{Frequency response during} noise stimuli

To investigate the frequency tuning of PNs using more naturalistic input waveforms, we measured the frequency-dependent gain, $G(f)$, and the mean phase shift, $\phi(f)$, of the spike response with respect to each frequency component of a noise stimulus, $I_{\text {noise }}(t)$, which is referred to here as $s(t) . G(f)$ and $\phi(f)$ were measured by correlation analysis of $s(t)$ and the digitized firing rate, $r(t)$ (Lee and Schetzen, 1965; Guttman et al., 1974; Bryant and Segundo, 1976) (see Materials and Methods). The analysis is illustrated in Figure $4 A-E$, showing the results for an L2-3 PN stimulated with $5 \mathrm{~ms}$ exponential-filtered noise, as described above. The stimulus-response correlation, $c_{\mathrm{sr}}(\tau)$ (Fig. $4 B$, solid line), is related to the spike-triggered average current, where $I_{\mathrm{STA}}(\tau)=c_{\mathrm{sr}}(-\tau) / r_{0}$ (Bryant and Segundo, 1976). $G(f)$ (Fig. 4C) and $\phi(f)$ (Fig. 4D) were obtained by Fourier analysis of the two correlations (see Materials and Methods). It is apparent that $\phi(f)$ shows a large phase lag (positive value) at high frequencies. Much of this lag can be accounted for by a fixed time delay between stimulus current fluctuations and detected spikes. This delay was estimated as the location of the peak of $c_{\mathrm{sr}}(\tau)(0.5 \mathrm{~ms}$ in the illustrated example), and the resulting phase lag was subtracted to obtain the corrected $\phi(f)$ (Fig. $4 E$ ). The average $G(f), \phi(f)$, and corrected $\phi(f)$ in 13 L2-3 PNs stimulated with $5 \mathrm{~ms}$ exponential-filtered noise and firing at $\sim 10 \mathrm{~Hz}$ (Fig. $4 F-H$ ) show similar features to the example cell.

These results confirm previous findings that cortical PNs can effectively transmit high-frequency stimulus fluctuations (Carandini et al., 1996; Köndgen et al., 2008). In most cells, $G(f)$ did not fall off at frequencies up to $\sim 400 \mathrm{~Hz}$ and declined by less than half at $1000 \mathrm{~Hz}$. The true falloff may be less, because the measured decline may result in part from technical limitations. Above 300 $\mathrm{Hz}, G(f)$ decreased somewhat when the pipette capacitance compensation was turned off. Because capacitance cannot be compensated completely, the unavoidable undercompensation may lead to underestimation of $G(f)$ at the upper end of the frequency 


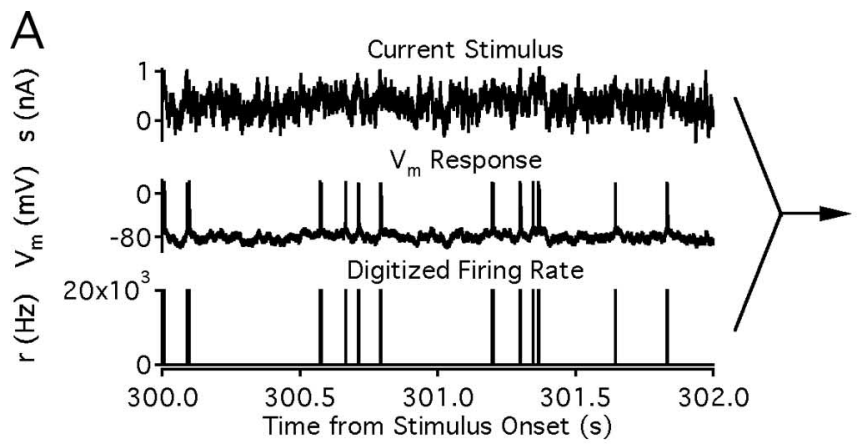

C
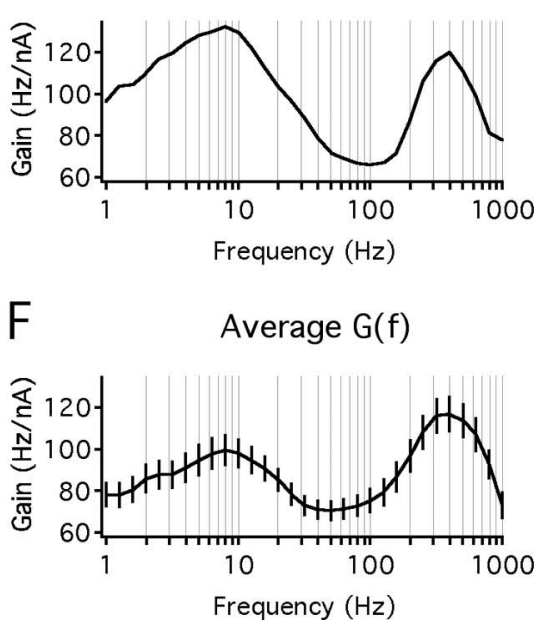

D

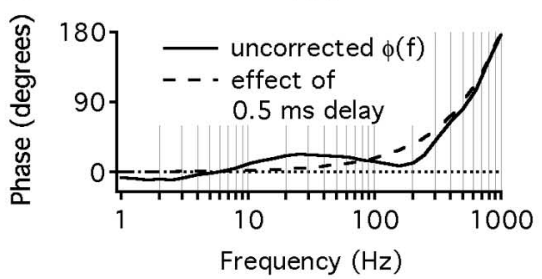

G

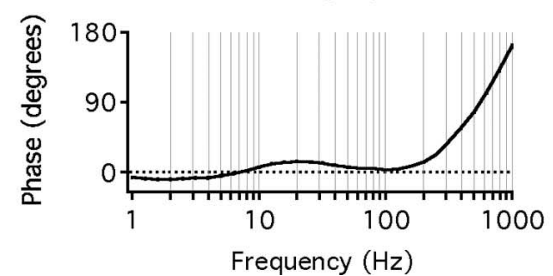

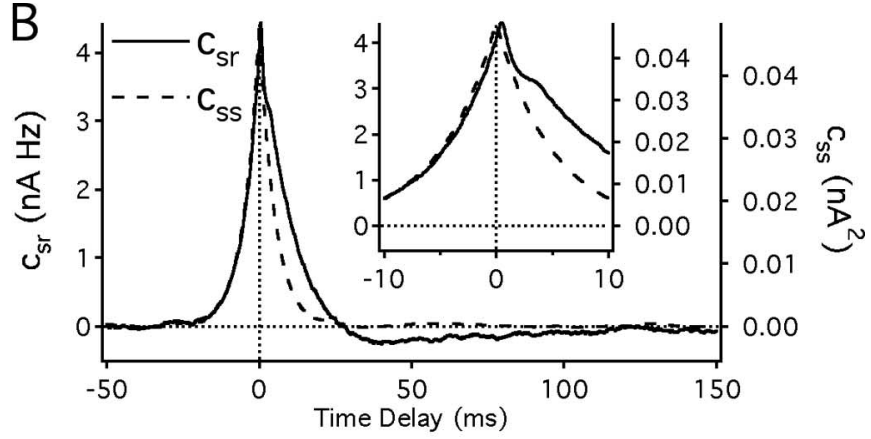

$\mathrm{E}$
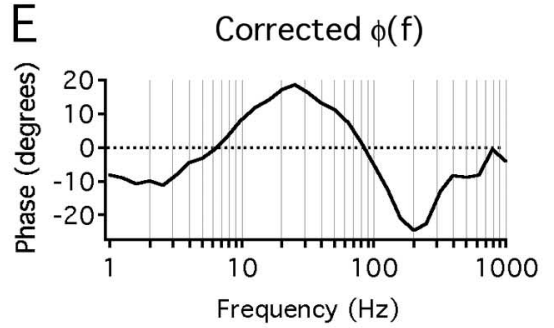

$\mathrm{H} \quad$ Average Corrected $\phi(\mathrm{f})$

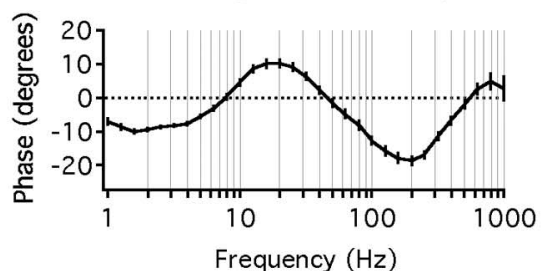

Figure 4. Measuring the gain of $L 2-3$ PNs over a wide range of frequencies. $A$, Example of current stimulus (top), $V_{m}$ response (middle), and digitized firing rate (bottom) in a neuron stimulated with $5 \mathrm{~ms}$ exponential-filtered noise. $\boldsymbol{B}$, Correlation of the current stimulus with the digitized firing rate $\left(\boldsymbol{c}_{\mathrm{sr}}\right)$ (solid line, scale on left axis) and autocorrelation of the stimulus ( $\left.\boldsymbol{c}_{\mathrm{ss}}\right)$ (dashed line, scale on right axis). Inset shows the same correlations on an expanded timescale. The peak of $c_{\mathrm{sr}}$ was found at a delay of 0.5 ms. $C$, Frequency-dependent gain, $G(f)$, calculated based on $c_{\mathrm{sr}}$ and $c_{\mathrm{ss}}$, with frequency-dependent windowing (see Materials and Methods). $D$, Mean phase of response, $\phi(f)$ (solid line). Dashed line shows the phase delay caused by the 0.5 ms time delay estimated from the peak of $c_{\mathrm{sr}}, \boldsymbol{E}$, Mean phase corrected for the delay. $\boldsymbol{F}$, Average $G(f)$ in seven $\mathrm{L} 2-3$ PNs stimulated with 5 ms exponential-filtered noise, with I adjusted to give $r_{0} \approx 10$ Hz. $\boldsymbol{G}$, Average uncorrected $\phi(f)$ in these cells. $\boldsymbol{H}$, Average $\phi(f)$ corrected for the estimated time delay of each neuron.

range analyzed. The high gain of $\mathrm{L} 2-3 \mathrm{PNs}$ at high $f$ does not imply that a single neuron can encode a high-frequency input; this is limited by the relatively low $r_{0}$. Instead, this result suggests that a population of PNs with common input can transmit highfrequency signals. A rapid sinusoidal modulation of firing probability appears as a slight effect on spike timing in each individual neuron but translates directly to a sinusoidal modulation of the average population firing rate (Fourcaud-Trocmé et al., 2003).

$G(f)$ shows two peaks. In cells stimulated with $5 \mathrm{~ms}$ exponential-filtered noise, the first peak, which we refer to as the "primary resonance," was located at $8.7 \pm 2.4 \mathrm{~Hz}$, falling slightly below the mean firing rate of $9.95 \pm 0.49 \mathrm{~Hz}$. The second peak, which we call the "high-frequency resonance," was found at $422 \pm 95 \mathrm{~Hz}$, which corresponds approximately to the highest intraburst firing rates observed. Thus, both resonances may have some relationship to the ISIs of single L2-3 PNs. However, a high-frequency resonance was seen in bursting and nonbursting neurons, indicating that correspondingly short ISIs are not required for its generation. Experiments described below investigated the influence of burst firing on the two resonance peaks.

The corrected $\phi(f)$ (Fig. $4 E, H$ ) also has features indicating that two resonant frequencies are present. At low $f$, phase leads (negative values) are observed; because of spike frequency adaptation, the highest firing probability occurs on the rising phase of slow stimulus fluctuations (Benda and Herz, 2003; Lundstrom et al., 2008). At a frequency near the first peak of $G(f), \phi(f)$ crosses zero. A rapid phase change near the resonant frequency is characteristic of many resonant systems, and the zero crossing was used previously as a measure of the preferred frequency (Fuhrmann et al., 2002). Above $\sim 50 \mathrm{~Hz}$, phase leads are again seen, and a shift toward phase lag is found near the high-frequency peak of $G(f)$, consistent with a second resonance.

\section{Frequency response for different stimulus waveforms}

Because neuronal spike generation is nonlinear, $G(f)$ depends on the statistics of the stimulus. For example, studies have shown that the "steady-state" (low-frequency) gain of neurons depends on the SD of stimulus noise (Chance et al., 2002; Longtin et al., 2002; Fellous et al., 2003; Shu et al., 2003; Higgs et al., 2006; Arsiero et al., 2007). Here, we investigated how frequencydependent gain depends on the stimulus power spectrum, measuring $G(f)$ in response to $1 \mathrm{~ms}$ exponential-filtered noise and $1 / f$ noise, in addition to the $5 \mathrm{~ms}$ exponential-filtered noise described above. The $1 \mathrm{~ms}$ noise may be thought of as simulating very fast synaptic currents, whereas $1 / f$ noise contains fluctuations spanning multiple timescales, a property that is characteristic of some sensory stimuli (Voss and Clarke, 1975; van Hateren, 1997) and forms of neural activity (Teich et al., 1997; Mazzoni et al., 2007). $1 / f$ noise is also useful as an experimental tool because its substantial power at low frequency allows one to measure gain over a 


\section{A}

1 ms Exponential Noise
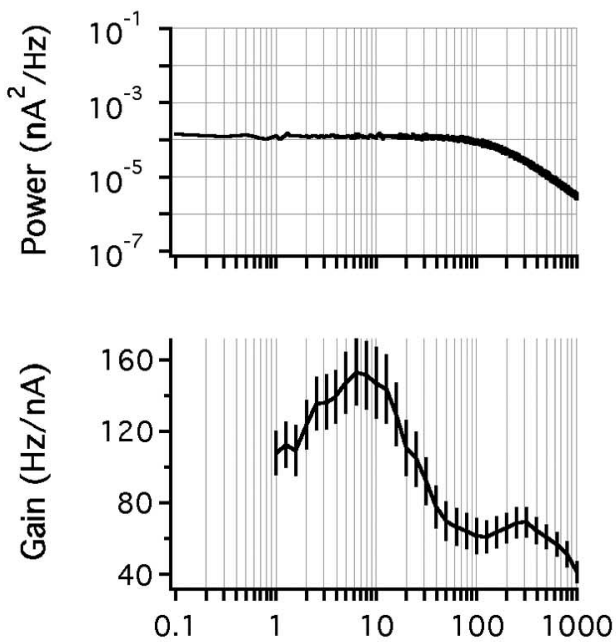

$\mathrm{B}$
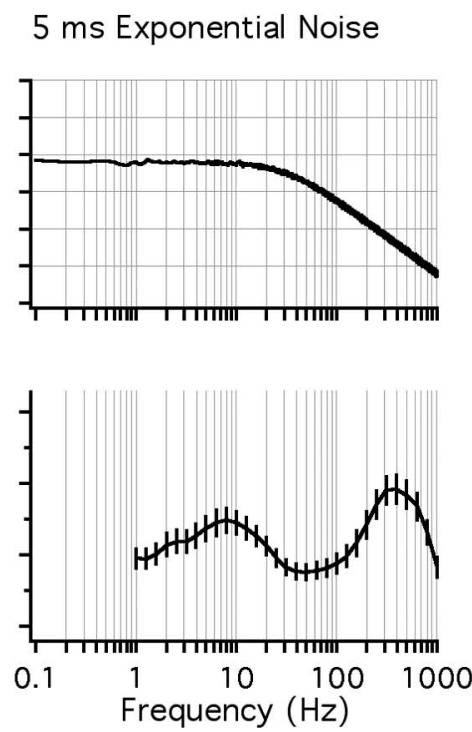

$1 / f$ Noise
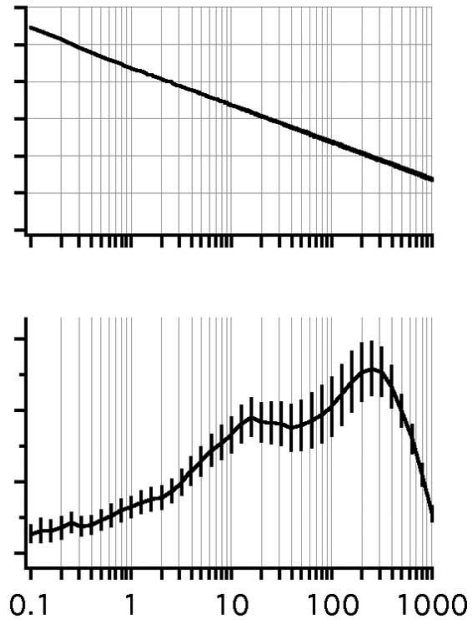

\section{Primary Resonance}

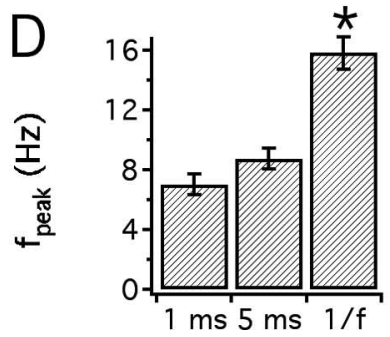

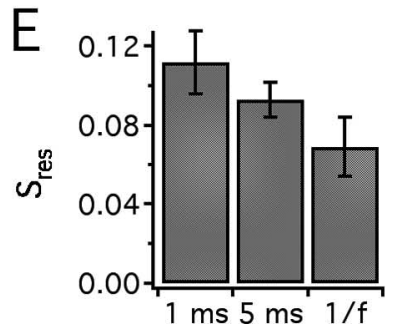

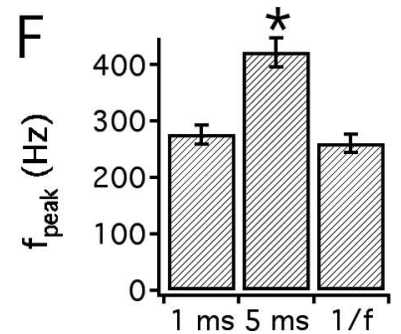

High-Frequency Resonance

Figure 5. Gain-frequency relationships of $\mathrm{L} 2-3$ PNs depend on the stimulus. $A-C$, Top row shows the power spectrum of each noise stimulus for $\sigma_{l}=0.25 \mathrm{nA}$. Bottom row shows average $G(f)$, with $I_{D C}$ and/or $\sigma_{l}$ adjusted to obtain $r_{0} \approx 10 \mathrm{~Hz}$. D, Peak frequency of primary resonance, showing a higher value for $1 / f$ noise. Asterisks in $\boldsymbol{D}, \boldsymbol{F}$, and $\boldsymbol{G}$ indicate that there was a significant difference between stimuli, based on a single-factor ANOVA, and the measure for the indicated stimulus differed significantly from the corresponding measure for both other stimuli, based on post hoc $t$ tests with Bonferroni's correction factor. $\boldsymbol{E}$, Strength of primary resonance. $\boldsymbol{F}$, Peak frequency of high-frequency resonance, showing a higher value for $5 \mathrm{~ms}$ exponential-filtered noise. $\boldsymbol{G}$, Strength of high-frequency resonance, which was also greatest for 5 ms exponential-filtered noise. ${ }^{*} p 0.05$.

wider frequency range. All noise stimuli were added to $I_{\mathrm{DC}}$ slightly below the rheobase, and the stimulus parameters $\left(\sigma_{I}\right.$ and/or $I_{\mathrm{DC}}$ ) were adjusted to give $r_{0} \approx 10 \mathrm{~Hz}$.

The power spectra of the three stimuli are shown in Figure $5 A-C$ (top panels), comparing noise of equal $\sigma_{I}(0.25 \mathrm{nA})$. The average $G(f)$ in L2-3 PNs subjected to each stimulus is illustrated in the bottom panels ( $1 \mathrm{~ms}$ noise, $n=8 ; 5 \mathrm{~ms}$ noise, $n=13 ; 1 / f$ noise, $n=11)$. In all cases, two peaks were observed. However, the shape of the $G(f)$ curve clearly depends on the stimulus power spectrum. In general, the faster-fluctuating stimulus ( $1 \mathrm{~ms}$ exponential noise) gave higher gain at low $f$ and lower gain at high $f$ compared with a slower stimulus of the same type (5 ms exponential noise).

To quantify each resonance, we measured the peak frequency $\left(f_{\text {peak }}\right)$ and an index of resonance strength, or peak sharpness $\left(S_{\text {res }}\right)$. For the primary resonance, $f_{\text {peak }}$ was $7.0 \pm 1.9 \mathrm{~Hz}$ for $1 \mathrm{~ms}$ noise, $8.7 \pm 2.4 \mathrm{~Hz}$ for $5 \mathrm{~ms}$ noise, and $15.8 \pm 3.2 \mathrm{~Hz}$ for $1 / f$ noise (Fig. $5 D$ ). The higher $f_{\text {peak }}$ observed for $1 / f$ noise may result from the different firing pattern elicited by this stimulus. The slow fluctuations present in $1 / f$ noise caused long runs of firing at relatively high rate, separated by long quiet periods at low rate. Thus, most spikes occurred during periods of relatively fast firing. If the resonance depends primarily on regularities in the pattern of spikes and associated AHPs and ADPs, this pattern would be expected to increase the resonant frequency. The strength $\left(S_{\text {res }}\right)$ of the primary resonance did not differ significantly between stimuli but showed a trend toward higher values for $1 \mathrm{~ms}$ noise and lower values for $1 / f$ noise (Fig. $5 E$ ). For the high-frequency resonance, $f_{\text {peak }}$ was $276 \pm 49 \mathrm{~Hz}$ for $1 \mathrm{~ms}$ noise, $422 \pm 95 \mathrm{~Hz}$ for $5 \mathrm{~ms}$ noise, and $260 \pm 55 \mathrm{~Hz}$ for $1 / f$ noise (Fig. $5 F$ ). The strength of the high-frequency resonance was greatest for $5 \mathrm{~ms}$ noise (Fig. $5 G)$.

These results indicate that the suprathreshold frequency response of L2-3 PNs is nonlinear, because $G(f)$ varies substantially with changes in the stimulus waveform. Gain at low frequencies is higher when the stimulus contains less power at low $f$, and gain at high frequencies is greater when the input has less power at high $f$. This nonlinearity may promote transmission of relatively weak frequency components of the synaptic input, providing a more balanced frequency representation in the spike output. Transmission of weak high-frequency inputs may be biologically useful, because high-frequency signal components are required for precise encoding of rapid changes in sensory input and motor commands but are attenuated by the low-pass effects of synapses and dendrites. A nonlinear boosting effect may help to preserve high-frequency signals, particularly when these are weak (e.g., when the synaptic input arrives on distal dendrites).

\section{Contribution of burst spikes to the frequency response}

To investigate whether bursting contributes to the two resonances, we divided spikes elicited by noise stimulation into "burst spikes" (those preceded and/or followed by an ISI $<10 \mathrm{~ms}$ ) and 
A

$r(t)$

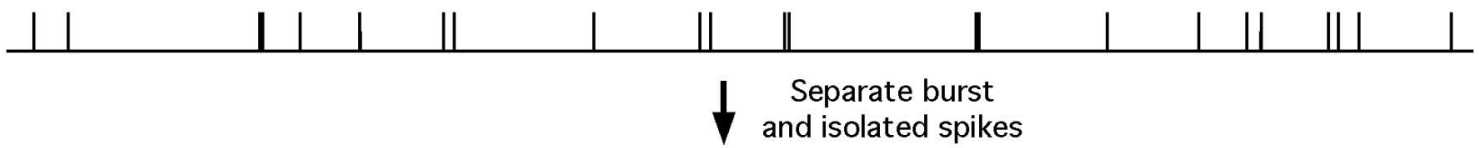

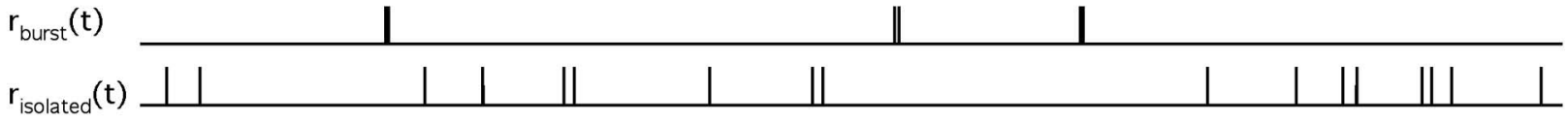

B

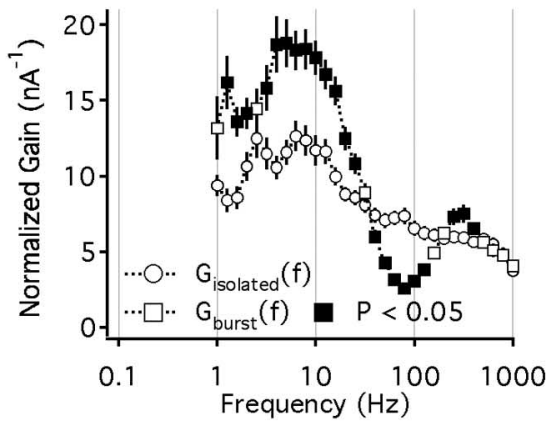

E
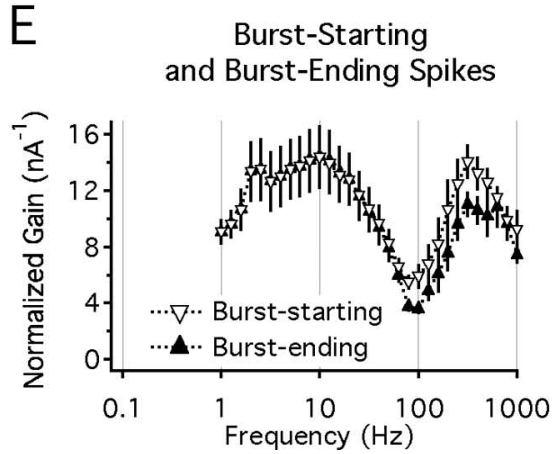

C $5 \mathrm{~ms}$ Exponential-Filtered Noise

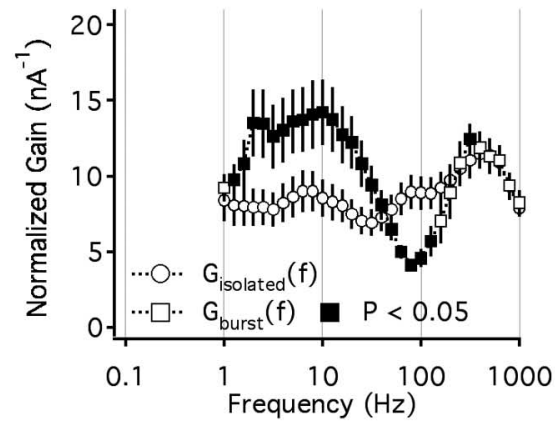

$\mathrm{F}$

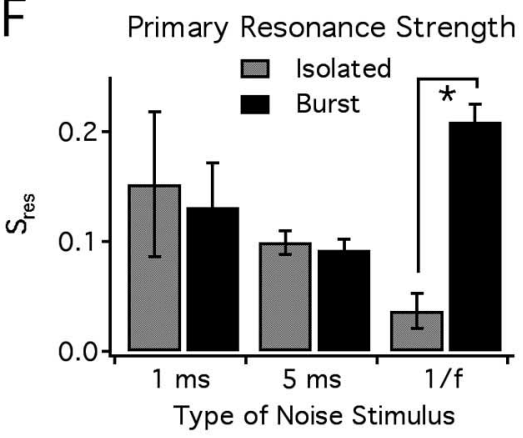

D $\quad 1 / f$ Noise

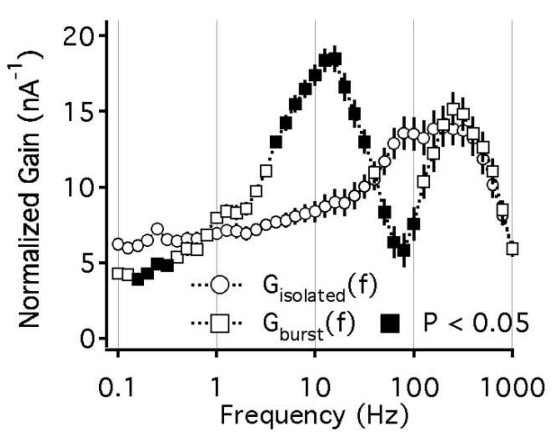

G High-Frequency Resonance Strength

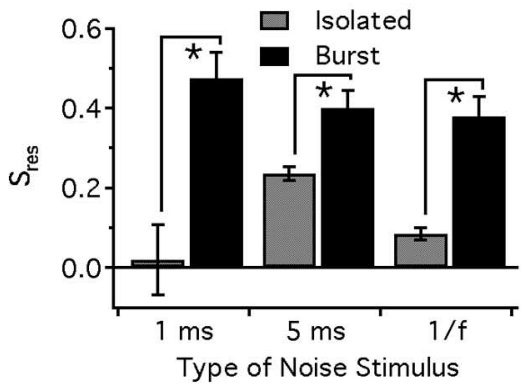

Figure 6. Gain analysis for burst and isolated spikes. $A$, Separate digitized firing rates, $r_{\text {burst }}(t)$ and $r_{\text {isolated }}(t)$, were constructed for burst and isolated spikes. $G_{\text {burst }}(f)$ and $G_{\text {isolated }}(f)$ were calculated based on the correlation between each component response and the stimulus current and were normalized by the mean rate of each subset of spikes. All data shown were obtained with stimuli adjusted to give $r_{0} \approx 10 \mathrm{~Hz}$, in cells that fired $>15 \%$ of their spikes in bursts. $\boldsymbol{B}$, Average $G_{\text {burst }}(f)$ and $G_{\text {isolated }}(f)$ in response to 1 ms exponential-filtered noise $(n=6$ cells). $\boldsymbol{C}$, Five millisecond exponential-filtered noise $(n=10)$. $\boldsymbol{D}, 1 / f$ noise $(n=6)$. Unlike the other stimuli, $1 / f$ noise allowed reliable measurements of $G(f)$ at frequencies down to $0.1 \mathrm{~Hz}$. $\boldsymbol{E}$, Average $G(f)$ for burst-starting and burst-ending spikes, in response to $5 \mathrm{~ms}$ exponential-filtered noise. $\boldsymbol{F}$, Strength of primary resonance for isolated and burst spikes, for each type of noise. $\boldsymbol{G}$, Strength of high-frequency resonance, showing a significantly greater value for burst spikes compared with isolated spikes for each stimulus. ${ }^{*} p<0.05$.

"isolated spikes" (all others). Each neuron was classified as bursting or nonbursting based on whether the fraction of burst spikes exceeded the expected value for a Poisson process with an absolute refractory period of $2 \mathrm{~ms}$. With a mean firing rate of $10 \mathrm{~Hz}$, $\sim 15 \%$ of the Poisson spikes fall into the burst category. Thus, neurons with $>15 \%$ burst spikes were classified as bursting. This criterion is conservative because the ISI distributions of real nonbursting neurons show relative as well as absolute refractoriness, reducing the percentage of burst spikes. Subsequent analysis was restricted to bursting cells: 6 of 8 L2-3 PNs stimulated with $1 \mathrm{~ms}$ exponential noise, 10 of 13 with $5 \mathrm{~ms}$ exponential noise, and 5 of 11 with $1 / f$ noise.

Digitized firing rates, $r_{\text {burst }}(t)$ and $r_{\text {isolated }}(t)$, were constructed for burst spikes and isolated spikes (Fig. 6A), and $G_{\text {burst }}(f)$ and $G_{\text {isolated }}(f)$ were computed based on the correlation of each response component with the noise stimulus. The rates of burst and isolated spikes were generally different. If the frequencydependent modulation of relative spike probability were equal for the two sets of spikes, each gain curve would scale with the rate. Thus, for comparison, $G_{\text {burst }}(f)$ and $G_{\text {isolated }}(f)$ were nor- malized by the corresponding mean rates. The normalized gain has units of $\mathrm{nA}^{-1}$ and represents the relative modulation of firing probability per nanoampere of sinusoidal current.

$G_{\text {burst }}(f)$ and $G_{\text {isolated }}(f)$ were measured for cells stimulated with $1 \mathrm{~ms}$ exponential-filtered noise (Fig. $6 B$ ), $5 \mathrm{~ms}$ exponentialfiltered noise (Fig. 6C), and $1 / f$ noise (Fig. 6D). Although the shapes of the curves varied between stimuli, the differences between burst and isolated spikes were consistent. In each case, burst spikes provided higher gain around the primary resonance peak and lower gain between the two peaks. Surprisingly, the measured strength $\left(S_{\text {res }}\right)$ of the primary resonance (Fig. $\left.6 F\right)$ did not differ between isolated and burst spikes with 1 and $5 \mathrm{~ms}$ noise, although burst spikes showed stronger resonance with $1 / f$ noise. This result indicates that bursts did not consistently produce a sharper resonance peak within the frequency range used to measure $S_{\text {res }}\left(0.5 f_{\text {peak }}\right.$ to $\left.2 f_{\text {peak }}\right)$. However, bursts did provide higher gain at the peak relative to more widely separated frequencies on either side. For the high-frequency peak, $S_{\text {res }}$ was higher for burst spikes compared with isolated spikes with all three noise stimuli (Fig. 6G). This was primarily a consequence of lower gain be- 

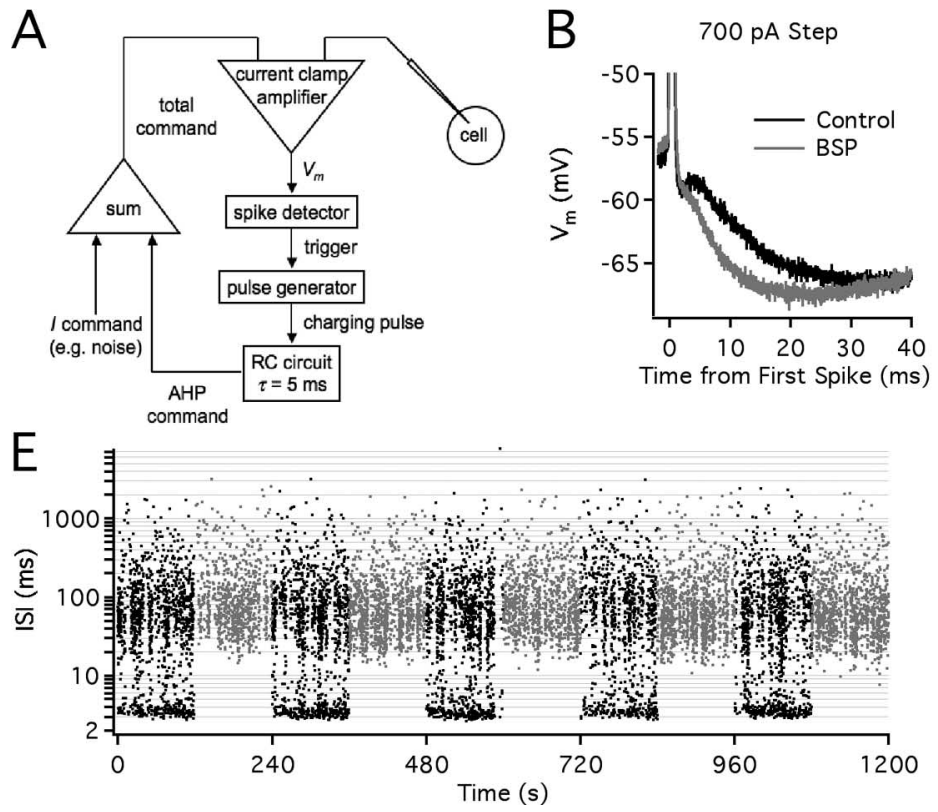
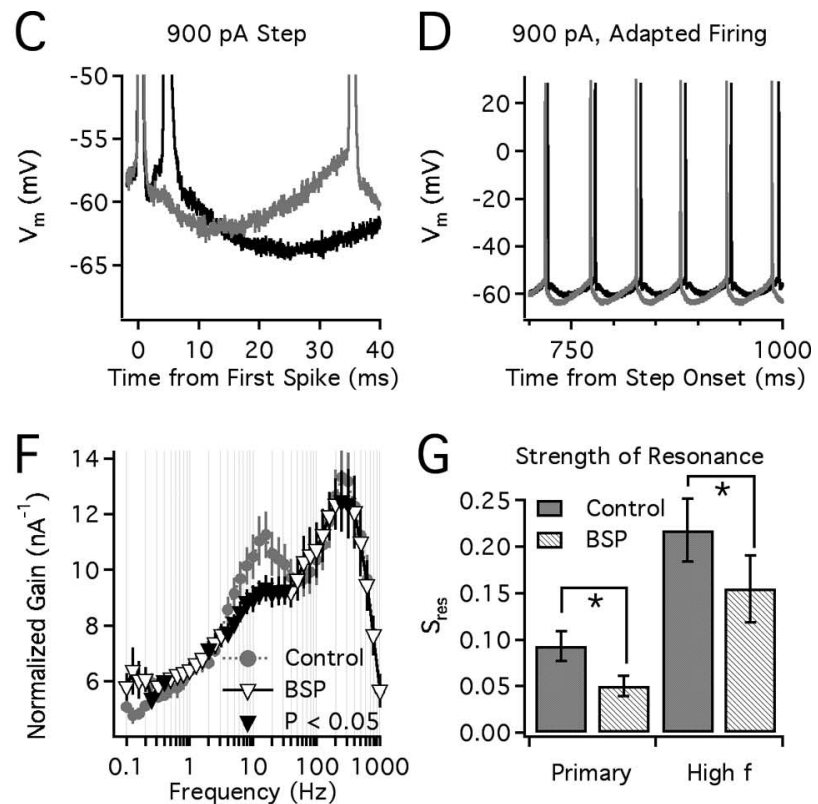

Figure 7. BSP by automated current feedback. $\boldsymbol{A}$, Schematic of the feedback circuit (see Materials and Methods). $\boldsymbol{B}$, Effect of BSP on the $V_{m}$ trajectory after a current step slightly above rheobase (700 pA). The fADP is truncated by a steeper descent into the mAHP. C, Effect of BSP on the initial firing to a larger step ( $900 \mathrm{pA}$ ). A two-spike burst is seen in the control response (black) but not with BSP (gray). D, Adapted firing ( $900 \mathrm{pA}$ step, $700-1000 \mathrm{~ms})$. BSP had little effect on the adapted firing rate. $\boldsymbol{E}$, Effect on ISIs during a $1 / \mathrm{f}$ noise stimulus $\left(I_{D C}=0.470 \mathrm{nA}, \sigma_{I}=0.225 \mathrm{nA}\right)$. Alternating 2 min stimulus blocks were presented as control (black dots) and with BSP (gray). The short ISIs centered at $3-4$ ms in control periods were eliminated by BSP. $F$, Average effect on $G(f)$ ( $n=5$ ). Filled black triangles indicate values obtained with BSP that were significantly different from the corresponding control data. $G$, Strength of primary and high-frequency resonance, showing significant reductions by $B S P .{ }^{*} p<0.05$.

tween the two peaks. For 1 and 5 ms exponential-filtered noise, we also observed slightly greater gain at the high-frequency peak.

The high $G_{\text {burst }}(f)$ around the primary resonance peak indicates that the probability of bursting was most strongly modulated by stimulus oscillations with periods of $\sim 100 \mathrm{~ms}$. The relatively low $G_{\text {burst }}(f)$ for slower oscillations was likely caused by adaptive mechanisms (Benda and Herz, 2003) (e.g., AHP currents, $\mathrm{Na}^{+}$channel inactivation, and Kv7 channel activation) that may build up during each stimulus elevation.

The low $G_{\text {burst }}(f)$ between the two peaks $(\sim 50-130 \mathrm{~Hz})$ suggests that intermediate-frequency stimulus oscillations do not strongly modulate the probability of burst firing. However, a low $G_{\text {burst }}(f)$ may also result directly from tightly distributed intraburst intervals. This may be appreciated by considering a scenario in which each burst interval is exactly $5 \mathrm{~ms}$. In this case, the probability of triggering the first spike of each burst may be strongly modulated by a $100 \mathrm{~Hz}$ sine wave current, but the gain at $100 \mathrm{~Hz}$ (and $300 \mathrm{~Hz}, 500 \mathrm{~Hz}, \ldots$ ) will be zero. The gain must be zero because the spike response, $r(t)$, has zero power at $100 \mathrm{~Hz}$, because the phase difference between each burst spike and its partner is exactly $180^{\circ}$.

To determine whether stereotyped intraburst intervals are primarily responsible for the low $G_{\text {burst }}(f)$ between the two resonance peaks, we computed $G(f)$ separately for burst-starting and burst-ending spikes. By removing the potentially out-ofphase partner spike, this analysis will reveal the features of $G_{\text {burst }}(f)$ that do not depend directly on the intraburst intervals. The results obtained with $5 \mathrm{~ms}$ exponential-filtered noise are illustrated in Figure $6 E$. The $G(f)$ curves for burst-starting and burst-ending spikes both show two peaks separated by a large dip with a minimum at $80-100 \mathrm{~Hz}$ (Fig. $6 E$ ). These data suggest that the low $G_{\text {burst }}(f)$ at $50-130 \mathrm{~Hz}$ does not arise primarily from tightly distributed intraburst intervals. Rather, oscillations in this frequency range are not effective at triggering two spikes separated by a short ISI.

\section{Burst suppression by automated current feedback}

The results described above indicate that burst and isolated spikes make different contributions to the two firing resonances. To investigate the effects of altered bursting on the overall frequency response without using potentially nonselective pharmacological agents, we applied burst suppression (BSP) by automated current feedback (Fig. 7A) (see Materials and Methods). On the down stroke of each detected spike, a pulse of hyperpolarizing current was added to the stimulus. Each pulse had a rise time of $0.2 \mathrm{~ms}$ and a peak of $-1 \mathrm{nA}$, and decayed exponentially with a time constant of $5 \mathrm{~ms}$. During test current steps slightly above the rheobase, BSP reduced the AADP and increased the steepness of the following AHP (Fig. 7B). When larger steps were applied, BSP prevented initial doublet firing (Fig. 7C) but had little effect on the adapted firing rate (Fig. $7 D$ ).

We next investigated the effect of BSP on bursting in response to $1 / f$ noise. Stimulus blocks $2 \mathrm{~min}$ in duration were applied, alternating five control blocks and five blocks with BSP. The effect on the firing pattern is illustrated by plotting the ISIs on a log axis, as a function of the total stimulus time (Fig. 7E). A band of short intervals is observed for each control period (black dots) but is absent for periods when BSP was applied (gray dots). In five L2-3 PNs, BSP reduced the percentage of spikes in bursts from $40 \pm 4$ to $3 \pm 2 \%(p=0.0005)$ but had relatively little effect on $r_{0}$ (control, $10.3 \pm 1.0 \mathrm{~Hz}$; BSP, $9.2 \pm 1.1 \mathrm{~Hz}$ ).

Finally, we calculated $G(f)$ for control and BSP blocks, normalizing by $r_{o}$ to correct for the small change that occurred (Fig. $7 F$ ). BSP lowered gain from 4 to $30 \mathrm{~Hz}$ and at the high-frequency peak $(\sim 250 \mathrm{~Hz})$ and reduced the strength of the primary and 


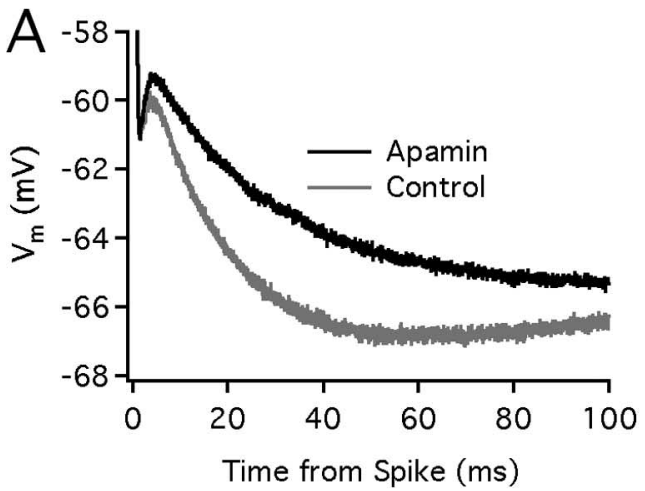

D Fraction of Spikes

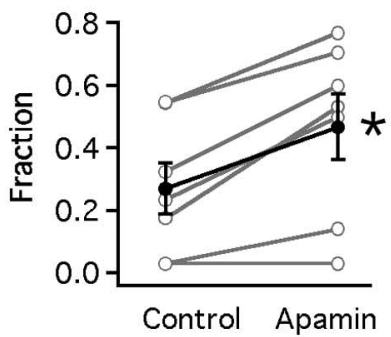

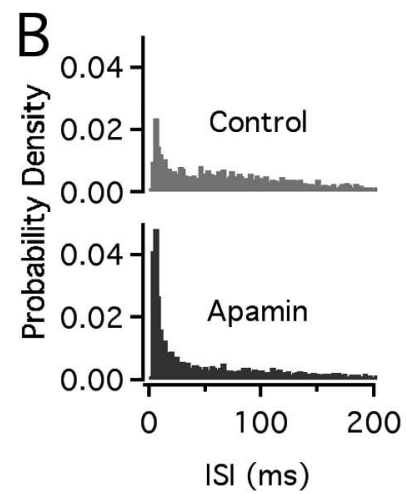

ISI (ms)

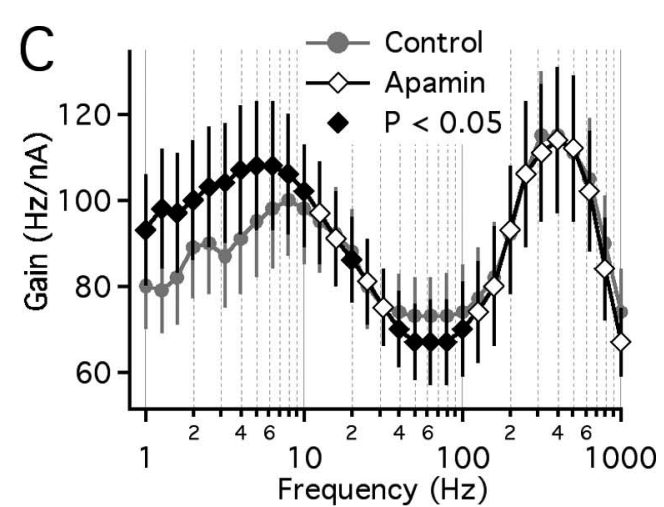

G Subthreshold Impedance Magnitude
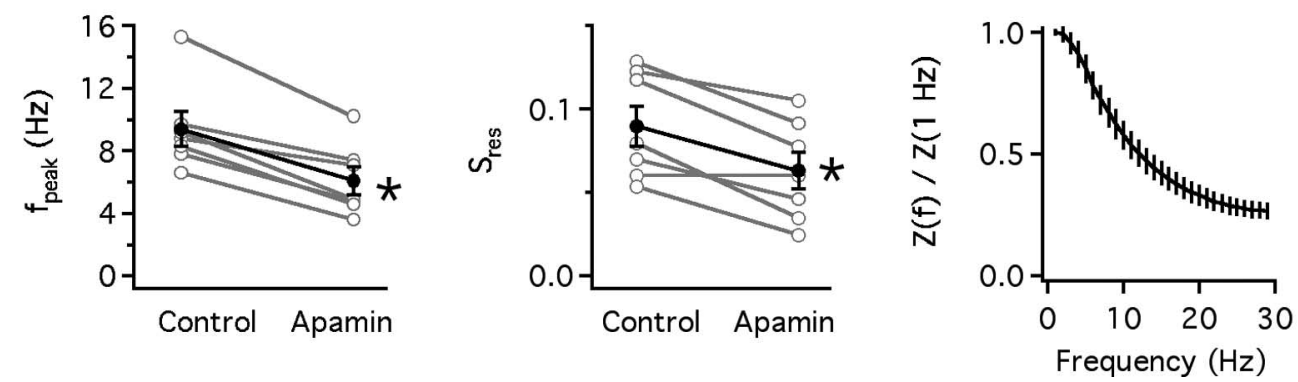

Figure 8. Effects of the SK channel blocker apamin on $G(f)$. $A$, Typical effect of apamin on the fADP and mAHP, illustrated by the $V_{m}$ trajectory after a single spike elicited by a 3 ms square current pulse added to $I_{D C}$ slightly below the rheobase. $B$, ISI histograms for a typical L2 -3 PN stimulated with $5 \mathrm{~ms}$ exponential-filtered noise $\left(\sigma_{I}=0.18 \mathrm{nA}\right)$ before and after wash in of apamin ( $\left.100 \mathrm{~nm}\right)$. In each case, $r_{0}$ was held at $\sim 10 \mathrm{~Hz}$ by adjusting $I_{D C}$. C, Average $G(f)$ in L2-3 PNs $(n=7)$ in control solution and in apamin, with $r_{0}$ controlled at $10 \mathrm{~Hz}$. $D$, Apamin increased the fraction of spikes in bursts. $\boldsymbol{E}$, Apamin reduced the primary resonance frequency. $\boldsymbol{F}$, Apamin reduced the strength of the primary resonance. $\mathbf{G}$, Frequency-dependent subthreshold impedance magnitude $(n=4)$, determined by injection of chirp current $(0-30 \mathrm{~Hz}$ in $60 \mathrm{~s})$ with $V_{m}$ set just below spike threshold by addition of $I_{\mathrm{DC}}$. Values are normalized by the impedance magnitude at $1 \mathrm{~Hz}$. ${ }^{*} p<0.05$.

high-frequency resonances (Fig. 7G). These results are consistent with the hypothesis that bursting enhances both resonances.

We note that the effect of BSP differs fundamentally from the previously described analytical treatment in which only the first or last spike of each burst was considered (Fig. 6E). The effect of BSP on the primary resonance depends on a change in the interburst intervals, which might result from the interaction of the burst mechanism and the mAHP. In control conditions, the large mAHP produced by each burst may regularize the output spike train, contributing to the primary resonance. BSP leaves only the smaller mAHPs that follow single spikes, which may account for the weakening of resonance. The studies described below tested the hypothesis that the SK channel-mediated mAHP contributes to resonant firing.

\section{Effects of the SK channel-mediated mAHP}

Although resonance can be amplified by a fast positive feedback mechanism such as the fADP, the primary requirement is negative feedback that is slow relative to the output dynamics (e.g., the membrane time constant for resonance of $V_{m}$ ) (Hutcheon and Yarom, 2000). For firing resonance at $\sim 10 \mathrm{~Hz}$, we hypothesized that the mAHP current mediated by small-conductance $\mathrm{Ca}^{2+}$ activated $\mathrm{K}^{+}$(SK) channels might contribute to this negative feedback. To test this hypothesis, we applied the SK blocker apamin $(100 \mathrm{nM})$, which has been shown to reduce the mAHP in rat L2-3 PNs (Abel et al., 2004). Typical effects of apamin on the fADP and $\mathrm{mAHP}$ are illustrated by the $V_{m}$ trajectory after a single spike elicited by a $3 \mathrm{~ms}$ current pulse, holding $I_{\mathrm{DC}}$ just below the rheobase (Fig. $8 \mathrm{~A}$ ). The drug causes a slight increase in the height of the AADP, followed by a slower descent into a smaller mAHP.
These results indicate that activation of SK channels normally limits the height and width of the fADP.

We measured the effects of apamin on burst firing and $G(f)$ in seven L2-3 PNs, using $5 \mathrm{~ms}$ exponential-filtered noise $\left(\sigma_{I}=\right.$ $0.28 \pm 0.10 \mathrm{nA}$ ). Because SK channels are known to control firing rate in neocortical PNs (Schwindt et al., 1988; Lorenzon and Foehring, 1992) and $G(f)$ depends on $r_{0}$, we held $r_{0}$ at $\sim 10 \mathrm{~Hz}$ throughout each experiment by automated adjustment of $I_{\mathrm{DC}}$ (see Materials and Methods). Apamin data were taken after this adjustment stabilized, showing complete wash in of the drug. On average, $I_{\mathrm{DC}}$ was lowered from $0.47 \pm 0.21$ to $0.39 \pm 0.22 \mathrm{nA}$, and $r_{0}$ was $10.2 \pm 0.2 \mathrm{~Hz}$ in control solution and $10.1 \pm 0.1 \mathrm{~Hz}$ in apamin. Despite the adjustment of $I_{\mathrm{DC}}$, apamin increased bursting in most cells (Fig. $8 B, D$ ), as expected based on the increased height and width of the fADP.

Analysis of $G(f)$ showed that apamin increased gain at 1-10 $\mathrm{Hz}$, reduced gain slightly at $40-100 \mathrm{~Hz}$, and had little effect at higher frequencies (Fig. 8C). Higher gain at low $f$ is consistent with previous studies showing that apamin increased gain determined from the steady-state firing rate at the end of long current steps (Schwindt et al., 1988; Lorenzon and Foehring, 1992) and increased stimulus-response coherence for low-frequency components of a noise stimulus (Ellis et al., 2007). Lower gain at $40-100 \mathrm{~Hz}$ most likely resulted from the greater fraction of spikes in bursts, based on our analysis of isolated and burst spikes described above. Apamin did not eliminate the primary resonance but lowered $f_{\text {peak }}$ (Fig. $8 E$ ) and reduced $S_{\text {res }}$ (Fig. $8 F$ ).

Apamin had no significant effect on the peak frequency or strength of the high-frequency resonance (data not shown). This result was surprising to us, because the BSP experiments de- 

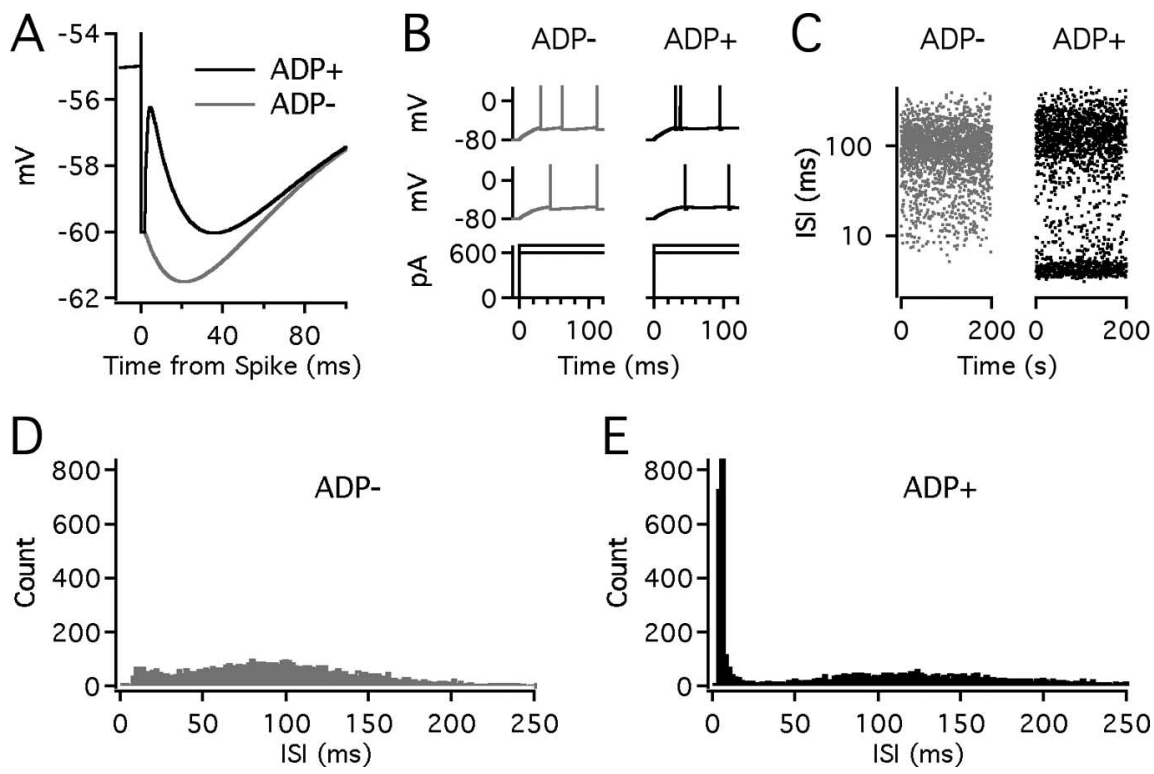

Figure 9. Conditional burst firing in a minimal model. Simulations were performed using an LIF model (see Materials and Methods). $A, V_{m}$ trajectory after the first spike during a just-suprathreshold current step ( $501 \mathrm{pA}$; rheobase, $\left.500 \mathrm{pA}\right)$ in models with and without a fast ADP, both including a medium AHP. After a $V_{m}$ reset (simulating the fAHP), the combination of ADP and AHP currents causes a rising and falling $V_{m}$ trajectory. $\boldsymbol{B}$, Responses to current steps (middle, $0.6 \mathrm{nA}$; top, $0.7 \mathrm{nA}$ ). The ADP ${ }^{+}$model (right) generates an initial two-spike burst during the $0.7 \mathrm{nA} \mathrm{step.}$. I ISIs in response to 5 ms exponential-filtered noise $\left(\sigma_{l}=0.25\right.$ $\mathrm{nA}, I_{\mathrm{DC}}=0.50 \mathrm{nA}$ ). The ADP ${ }^{+}$model produces two bands of ISIs, similar to L2 -3 PNs. D, ISI histogram for the ADP ${ }^{-}$model ( $2 \mathrm{~ms}$ bins) shows few short ISIs. $E$, ISI histogram for the ADP ${ }^{+}$model has a large peak of short ISIs and a second, broad peak of long intervals.

scribed above showed that manipulation of bursting altered the sharpness of this resonance. We considered the possibility that the lowering of $I_{\mathrm{DC}}$ required to maintain the target firing rate somehow prevented a change in high-frequency resonance, performing several control experiments $(n=4)$ in which $I_{\mathrm{DC}}$ was lowered by a similar amount without applying apamin. This reduced $r_{0}$ from $\sim 10$ to $\sim 5 \mathrm{~Hz}$ but had no consistent effect on the fraction of spikes in bursts. Lowering $I_{\mathrm{DC}}$ reduced the absolute gain at high frequencies but did not alter the shape of the highfrequency peak or the measured $S_{\text {res }}$ (data not shown). This result suggests that adjustment of $I_{\mathrm{DC}}$ is unlikely to have masked an effect of apamin on high-frequency resonance. This issue is considered further in Discussion.

Our data indicate that SK channels contribute to shaping the primary resonance. However, assuming that $100 \mathrm{nM}$ apamin was sufficient to block most of the SK channels, these results also suggest that additional negative feedback mechanisms capable of supporting resonant firing are present in L2-3 PNs. These may include slow AHP currents, $\mathrm{Na}^{+}$channel inactivation, and voltage-gated $\mathrm{K}^{+}$currents that may contribute to the mAHP.

An additional possibility is that firing resonance arises in part from subthreshold voltage-gated currents. To examine this, we measured the frequency-dependent membrane impedance, $Z(f)$, in L2-3 PNs $(n=4)$, holding $V_{m}$ just below spike threshold by addition of $I_{\mathrm{DC}}$ (see Materials and Methods). In these cells, $Z(f)$ decreased monotonically over the frequency range tested, from 0 to $30 \mathrm{~Hz}$ (Fig. 8G). Although extensive studies of this type were not performed, the results suggest that firing resonance in L2-3 PNs may be caused primarily by spike-dependent mechanisms.

\section{Bursting enhances firing resonance in a minimal model}

To explore the generality of the observed effects of bursting on $G(f)$ and to elucidate the minimal mechanism required, we per- formed simulations using singlecompartment LIF models with a spikedependent mAHP current, with and without a fast $\mathrm{ADP}$ current $\left(\mathrm{ADP}^{+}\right.$and $\mathrm{ADP}^{-}$, respectively). These models lack any subthreshold resonance, so any firing resonance depends entirely on the spikedependent currents. The model parameters (see Materials and Methods) were chosen to give $V_{\text {rest }}, \tau_{m}$, firing rates, and stimulus-dependent bursting similar to those of a typical L2-3 PN.

After a single spike during a justsuprathreshold current step, the $\mathrm{ADP}^{+}$ model generates a biphasic $V_{m}$ trajectory, whereas the $\mathrm{ADP}^{-}$model shows a monotonic descent into the mAHP (Fig. 9A). At the onset of sufficiently large current steps, the $\mathrm{ADP}^{+}$model generates an initial doublet followed by single spikes (Fig. 9B, top right). In response to noise, the $\mathrm{ADP}^{+}$ model produces a distinct subdistribution of short ISIs, in addition to a broad group of long intervals (Fig. 9C,E). In contrast, the $\mathrm{ADP}^{-}$model does not fire at short ISIs, either at the onset of current steps (Fig. 9B, left) or during a noise stimulus (Fig. 9C,D), unless the applied current is extremely large.

To investigate how burst firing affects the frequency response, we measured $G(f)$ based on responses of the nonbursting $\mathrm{ADP}^{-}$ model (Fig. 10A) and the bursting $\mathrm{ADP}^{+}$model (Fig. $10 \mathrm{~B}$ ) to 5 ms exponential-filtered noise $\left(\sigma_{I}=0.25 \mathrm{nA}\right)$. For each model, four levels of $I_{\mathrm{DC}}$ were chosen to give $r_{0} \approx 5,10,15$, and $20 \mathrm{~Hz}$ (blue, green, orange, and red curves, respectively). In each case, $G(f)$ showed a primary peak near or below $r_{0}$ (Fig. 10C). The $\mathrm{ADP}^{+}$model also produced a small high-frequency resonance at $\sim 250 \mathrm{~Hz}$, particularly when $r_{0}>10 \mathrm{~Hz}$. However, the highfrequency peak was much smaller than that of L2-3 PNs, and no high-frequency resonance was obtained in the nonbursting model. These differences between the models and recorded neurons likely arise because the models did not include fast ionic mechanisms such as Kv1 channels that are present in L2-3 PNs (Guan et al., 2006, 2007a) and have been shown to increase highfrequency suprathreshold resonance in neurons elsewhere in the brain (Slee et al., 2005).

Compared with the $\mathrm{ADP}^{-}$model, the $\mathrm{ADP}^{+}$model gave slightly lower $f_{\text {peak }}$ at each $r_{0}$ (Fig. 10C) and greater $S_{\text {res }}$ at $r_{0} \geq 10$ $\mathrm{Hz}$ (Fig. 10D). These results suggest that bursting driven by a fast ADP can increase firing resonance in a neuron with spikedependent negative feedback provided by a medium-duration AHP.

To confirm that the AHP was necessary for the resonance, simulations were performed using $\mathrm{ADP}^{-}$and $\mathrm{ADP}^{+}$models lacking the AHP current. The only spike-dependent negative feedback was a small $V_{m}$ reset (to $5 \mathrm{mV}$ below threshold) and an absolute refractory period of $2 \mathrm{~ms}$ (same parameters used in the simulations described above). In the absence of the AHP current, the ADP current does not produce a distinct bump in the postspike $V_{m}$ trajectory but simply causes a more rapid rise in $V_{m}$ for a short time after the refractory period.

The $G(f)$ curves of the $\mathrm{AHP}^{-}$models (Fig. 10E,F) are predominantly low pass, showing no primary resonance. The AHP ${ }^{-}$ 
A Non-Bursting Model (ADP-)

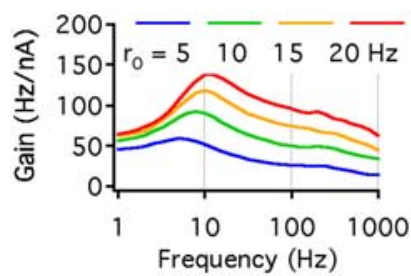

C

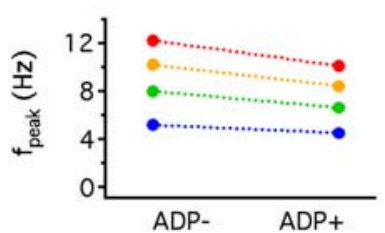

E Basic LIF Model (AHP- ADP-)

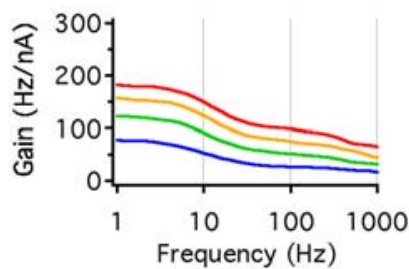

G Effect of Partial AHP Block
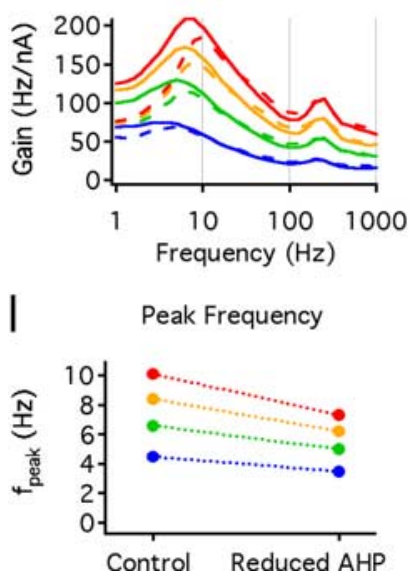

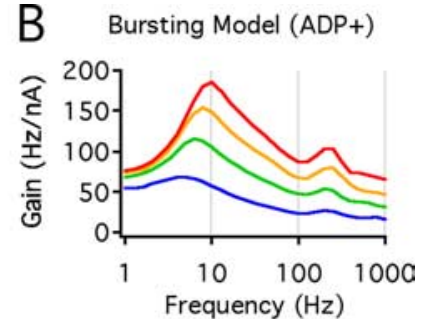

D Strength of Resonance

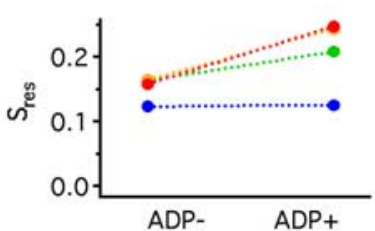

$\mathrm{F}$

AHP- ADP+ Model

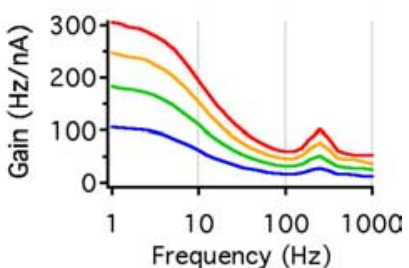

$H$ Fraction of Spikes in Bursts

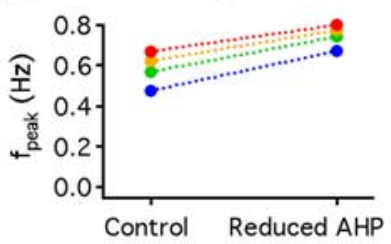

J Strength of Resonance

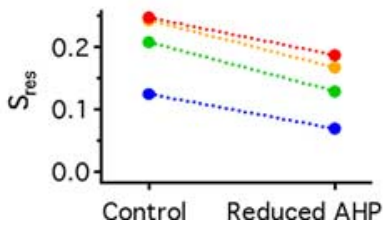

Figure 10. Bursting amplifies firing resonance in a minimal model. Simulations were performed with $5 \mathrm{~ms}$ exponential-filtered noise $\left(\sigma_{l}=0.25 \mathrm{nA}\right) . \boldsymbol{A}, G(f)$ for the nonbursting $\left(\mathrm{ADP}^{-}\right)$model. Here and in subsequent panels, levels of $I_{D C}$ were chosen to give $r_{0}=5,10,15$, and $20 \mathrm{~Hz}$ (blue, green, orange, and red, respectively). $\boldsymbol{B}, G(f)$ for the bursting (ADP ${ }^{+}$) model, showing higher gain at the primary peak and a small high-frequency resonance at $\sim 250 \mathrm{~Hz}$. $C$, At each $r_{0}$, the primary resonant frequency $\left(f_{\text {peak }}\right)$ was slightly lower in the bursting model. $\boldsymbol{D}$, The strength of the primary resonance $\left(S_{\text {res }}\right)$ was higher in the bursting model for $r_{0}=10,15$, and $20 \mathrm{~Hz} . \boldsymbol{E}, G(f)$ for basic LIF model (AHP ${ }^{-}$ADP $^{-}$), showing low-pass behavior. $F$, In the absence of an AHP current, adding the ADP current increased gain at low $f$ and created a small high-frequency resonance. $\mathbf{G}$, Effect of reducing the AHP current by half. Control responses (dashed lines) are from the bursting model illustrated in $\boldsymbol{B}$. $\boldsymbol{H}$, Reducing the AHP increased the fraction of spikes in bursts. $I$, Reducing the AHP lowered the primary resonant frequency at each $r_{0} . J$, Reducing the AHP decreased the strength of the primary resonance at each $r_{0}$.

$\mathrm{ADP}^{+}$model has a high-frequency peak at $\sim 250 \mathrm{~Hz}$, but this remains much smaller than the high-frequency resonance of L2-3 PNs. The absence of a peak at $f \leq r_{0}$ confirms that the AHP current (or another slow negative feedback mechanism) is necessary for the primary resonance. Without the AHP current, the ADP current greatly enhances gain at low frequencies (Fig. 10,

compare $E, F)$. In contrast, when the AHP is present, the ADP increases gain only slightly at $f \ll r_{0}$, and its effect is greatest near $f_{\text {peak }}$ (Fig. 10, compare $A, B$ ).

The simulated effects of completely removing the AHP differ from our experimental results obtained using the SK channel blocker apamin, which altered but did not eliminate the primary resonance. A likely explanation is that SK channels carry only part of the mAHP current, and apamin does not affect other slow feedback mechanisms present in L2-3 PNs (e.g., slow AHPs, $\mathrm{Na}^{+}$ channel inactivation, and $\mathrm{M}$ current). Thus, we also simulated partial blockade of the AHP, reducing the conductance triggered by each spike from 5 to $2.5 \mathrm{nS}$. The resulting $G(f)$ curves are shown in Figure $10 G$ (solid lines), along with the corresponding data for the standard bursting model (dashed lines). It is apparent that reducing the AHP enhanced gain for $f<r_{0}$.

The effect of the simulated partial block of the AHP was somewhat complex because of the interaction between the ADP and the AHP. Reducing the AHP current increases the height and width of the ADP (the effect of apamin shown in Fig. $8 \mathrm{~A}$ ) and thereby increases the fraction of spikes in bursts (Fig. $10 \mathrm{H}$ ). This may account in part for a lowering of $f_{\text {peak }}$ (Fig. 10I), similar to the effect of adding ADP current. However, reducing the AHP decreased $S_{\text {res }}$ (Fig. 10J). This effect cannot be explained by the increase in bursting but results directly from the loss of negative feedback that controls gain at low frequencies.

In summary, the effects of reducing the AHP in the model are similar to those of apamin in L2-3 PNs. The model results indicate that spike-dependent AHP and ADP currents can generate and amplify a primary firing resonance similar to that of L2-3 PNs.

\section{Discussion}

We found that L2-3 PNs classified as RS based on firing to current steps generate single spikes and two-spike bursts in response to fluctuating input. Bursting enhances the primary firing resonance and can also sharpen a high-frequency resonance.

\section{The spectrum of bursting neurons}

The bursts observed in the present study resemble those of L2-3 chattering cells (Gray and McCormick, 1996; Brumberg et al., 2000). Unlike L5 intrinsically bursting (IB) neurons (Connors et al., 1982), which often fire bursts of three to six spikes, chattering cells fire single spikes or doublets during just-suprathreshold current steps and add spikes to each burst when the current is increased. The burst pattern reflects the underlying mechanism. In IB neurons, bursts are driven by $\mathrm{Ca}^{2+}$-dependent dendritic spikes (Helmchen et al., 1999; Williams and Stuart, 1999). In contrast, bursts in chattering cells do not require $\mathrm{Ca}^{2+}$ current but depend on persistent $\mathrm{Na}^{+}$current, which contributes to a brief fADP (Brumberg et al., 2000). Chattering cells may overlap with RS PNs in L2-3. RS and chattering cells have similar morphology (Nowak et al., 2003), and many RS cells burst during ionic and pharmacological manipulations (Brumberg et al., 2000; Traub et al., 2003). The present results show that RS PNs in L2-3 burst in response to fluctuating stimuli without any nonphysiological treatments.

A difference between RS neurons and dedicated chattering cells is the length of intraburst intervals: $\sim 3-5 \mathrm{~ms}$ in our RS neurons compared with $\sim 2-2.5 \mathrm{~ms}$ in chattering cells (Nowak et al., 2003). Shorter ISIs should promote resonance at higher frequencies, consistent with suggestions that chattering cells support gamma (25-70 Hz) oscillations (Gray and McCormick, 1996; Wang, 1999; Cunningham et al., 2004). Our findings sug- 
gest that the looser bursts of L2-3 RS neurons are not triggered efficiently by $50-130 \mathrm{~Hz}$ stimuli. Thus, bursting in RS cells may support slower oscillations.

\section{Resonance in cortical PNs and the role of bursting}

Our results show that L2-3 PNs have two firing resonances, and both can be enhanced by bursting. The SK channel-dependent mAHP also contributes to the primary resonance. Previous studies reported subthreshold resonance mediated by HCN channels, Kv7 channels, and persistent $\mathrm{Na}^{+}$current in several types of hippocampal and neocortical PNs (Gutfreund et al., 1995; Hutcheon et al., 1996; Leung and Yu, 1998; Hu et al., 2002; Peters et al., 2005). However, to our knowledge, subthreshold resonance has not been described in neocortical L2-3 PNs. We measured the frequency-dependent membrane impedance in a small sample of cells and did not find a peak (Fig. 8G), suggesting that spike-dependent mechanisms mediate the primary resonance. It remains possible that L2-3 PNs show subthreshold resonance for dendritic input, because studies of other PNs have shown that dendrites have strong subthreshold resonance caused by $I_{h}$ (Narayanan and Johnston, 2007).

Our observation that bursting can sharpen high-frequency resonance is consistent with previous observations in L5 PNs (Schindler et al., 2006). We found that high-frequency resonance was stronger for burst spikes than for isolated spikes, and BSP using automated current feedback weakened high-frequency resonance. However, substantial resonance remained in the absence of bursts. Surprisingly, apamin did not alter the high-frequency resonance, despite a large increase in bursting. It remains unknown why BSP altered high-frequency resonance but apamin did not. A possible explanation is that the BSP studies used $1 / f$ noise, causing resonance at $\sim 270 \mathrm{~Hz}$, whereas the apamin experiments used $5 \mathrm{~ms}$ exponential-filtered noise, giving a resonant frequency of $\sim 400 \mathrm{~Hz}$. The intraburst firing rate of $\sim 250 \mathrm{~Hz}$ may synchronize well with the resonance obtained with $1 / f$ noise but not with the faster resonance observed in response to $5 \mathrm{~ms}$ exponential noise.

Our results suggest that bursting can sharpen the highfrequency resonance under some conditions but is not its sole cause. Another potentially important mechanism of highfrequency resonance in PNs is a dynamic spike threshold (Azouz and Gray, 2000, 2003), which may arise from $\mathrm{Na}^{+}$channel inactivation and rapidly activating $\mathrm{K}^{+}$channels (Schwindt et al., 1988; Spain et al., 1991; Bekkers and Delaney, 2001; Guan et al., 2007a,b).

\section{Remaining questions about natural input}

The present results show that bursting in L2-3 PNs depends on large stimulus fluctuations, which are present in natural synaptic input (Destexhe and Paré, 1999). An important remaining question is how synaptic conductance will affect the firing patterns of these cells. In general, conductance reduces low-frequency $V_{m}$ excursions. This may inhibit bursting by reducing frequency components effective for burst triggering (e.g., $\sim 10 \mathrm{~Hz}$ ). Bursts have been observed in L2-3 PNs in vivo (de Kock and Sakmann, 2008), indicating that natural conductance does not preclude bursting. In this study, bursts similar to those reported here accounted for $\sim 15 \%$ of spikes in the awake state but were essentially absent under anesthesia. This result suggests that bursts in L2-3 PNs are enabled by activity or modulatory factors associated with alertness and may then contribute to functions including oscillatory synchronization, synaptic reliability (Lisman, 1997), and triggering of dendritic plateau potentials that regulate synaptic plasticity and dendritic integration (Larkum et al., 1999).

High synaptic conductance is also likely to affect the firing resonance of L2-3 PNs. In stellate cells of the entorhinal cortex, increasing conductance by dynamic clamp reduced subthreshold theta oscillations and spike clustering at theta frequency caused by the mAHP (Fernandez and White, 2008). Based on this and a possible reduction of bursting, we expect that the primary resonance of L2-3 PNs will be smaller in high-conductance states. Experiments to investigate this will require recently developed techniques for dynamic clamp using high-resistance electrodes (Brette et al., 2008), because we find that burst firing in L2-3 PNs is greatly weakened and AHPs are reduced by washout during whole-cell recording.

\section{Bursts and isolated spikes as channels of neuronal output}

We find that bursts and isolated spikes encode different frequency components of the simulated synaptic input and may thereby function as distinct output channels. Similar results were obtained in electrosensory pyramidal neurons of electric fish (Oswald et al., 2004). When these cells were stimulated with band-limited noise $(0-60 \mathrm{~Hz})$, bursts showed higher coherence with low stimulus frequencies, whereas isolated spikes had greater coherence with higher frequencies. Similarly, we find that $G_{\text {burst }}(f)$ is higher than $G_{\text {isolated }}(f)$ from $\sim 2$ to $30 \mathrm{~Hz}$ and lower from $\sim 30$ to $200 \mathrm{~Hz}$. Our results and theirs both suggest that bursts encode low-frequency input within the range from 2 to 60 $\mathrm{Hz}$. Our studies provide information about a wider frequency range, showing that burst spikes in L2-3 PNs do not provide higher gain below $1 \mathrm{~Hz}$ and show similar gain to isolated spikes above $\sim 300 \mathrm{~Hz}$.

To function as a channel of output, bursts must be detected selectively by postsynaptic targets. This may be enabled by shortterm synaptic facilitation (Lisman, 1997; Williams and Stuart, 1999). In the neocortex, excitatory synapses onto "lowthreshold-spiking" (LTS) interneurons show strong facilitation (Thomson et al., 1995; Thomson and Deuchars, 1997; Beierlein et al., 2003; Watanabe et al., 2005; Ali and Nelson, 2006; Ali et al., 2007). A few studies have reported facilitation at excitatory synapses onto fast-spiking (FS) interneurons (Thomson, 1997; Thomson and Deuchars, 1997), whereas several other studies showed depression at PN-FS connections (Beierlein et al., 2003; Watanabe et al., 2005; Ali and Nelson, 2006; Ali et al., 2007), and synapses from L2-3 PNs to other PNs in L2-3 or L5 generally depress (Williams and Atkinson, 2007). These findings suggest that signals encoded by bursts in L2-3 PNs may be transmitted selectively to LTS cells and perhaps to subtypes of FS cells.

\section{Implications for epilepsy}

Although the stimuli applied in the present studies are quite different from the strong synaptic input that $\mathrm{PNs}$ receive during seizures, our results are consistent with the idea that bursting in L2-3 PNs may contribute to epileptiform activity. Neocortical oscillations at $\sim 10 \mathrm{~Hz}$ are observed in vivo and in brain slices under conditions of enhanced excitability (Silva et al., 1991; Flint and Connors, 1996; Lukatch and MacIver, 1997; Steriade et al., 1998b; Castro-Alamancos, 2000, 2006; Castro-Alamancos and Rigas, 2002; Castro-Alamancos et al., 2007). Oscillations at $10 \mathrm{~Hz}$ occur while GABA receptors are blocked (Flint and Connors, 1996), suggesting that they are generated by the excitatory network. The present results suggest that bursting in L2-3 PNs may promote these oscillations by increasing gain at $\sim 10 \mathrm{~Hz}$. Consistent with this, blocking persistent $\mathrm{Na}^{+}$current, which reduces 
bursting in chattering neurons (Brumberg et al., 2000) and RS PNs (Traub et al., 2003), eliminated $10 \mathrm{~Hz}$ oscillations but preserved the primary spike-and-wave discharge (CastroAlamancos et al., 2007).

Bursting in L2-3 PNs may also contribute to fast ripple oscillations $(250-500 \mathrm{~Hz})$ that have been recorded in the neocortex of epileptic patients and may be involved in seizure initiation or propagation (Bragin et al., 1999; Worrell et al., 2004; Jirsch et al., 2006). By sharpening the high-frequency resonance, the bursting mechanism of L2-3 PNs may promote network synchronization at fast ripple frequency, particularly when strong synaptic input causes many spikes to occur in bursts or longer runs at high rates.

\section{References}

Abel HJ, Lee JC, Callaway JC, Foehring RC (2004) Relationships between intracellular calcium and afterhyperpolarizations in neocortical pyramidal neurons. J Neurophysiol 91:324-335.

Ali AB, Nelson C (2006) Distinct $\mathrm{Ca}^{2+}$ channels mediate transmitter release at excitatory synapses displaying different dynamic properties in rat neocortex. Cereb Cortex 16:386-393.

Ali AB, Bannister AP, Thomson AM (2007) Robust correlations between action potential duration and the properties of synaptic connections in layer 4 interneurones in neocortical slices from juvenile rats and adult rat and cat. J Physiol 580:149-169.

Arsiero M, Lüscher HR, Lundstrom BN, Giugliano M (2007) The impact of input fluctuations on the frequency-current relationships of layer 5 pyramidal neurons in the rat medial prefrontal cortex. J Neurosci 27:3274-3284.

Azouz R, Gray CM (2000) Dynamic spike threshold reveals a mechanism for synaptic coincidence detection in cortical neurons in vivo. Proc Natl Acad Sci U S A 97:8110-8115.

Azouz R, Gray CM (2003) Adaptive coincidence detection and dynamic gain control in visual cortical neurons in vivo. Neuron 37:513-523.

Beierlein M, Gibson JR, Connors BW (2003) Two dynamically distinct inhibitory networks in layer 4 of the neocortex. J Neurophysiol 90:2987-3000.

Bekkers JM, Delaney AJ (2001) Modulation of excitability by alphadendrotoxin-sensitive potassium channels in neocortical pyramidal neurons. J Neurosci 21:6553-6560.

Benda J, Herz AVM (2003) A universal model for spike-frequency adaptation. Neural Comput 15:2523-2564.

Bragin A, Engel J Jr, Wilson CL, Fried I, Buzsáki G (1999) High-frequency oscillations in human brain. Hippocampus 9:137-142.

Brette R, Piwkowska Z, Monier C, Rudolph-Lilith M, Fournier J, Levy M, Frégnac Y, Bal T, Destexhe A (2008) High-resolution intracellular recordings using a real-time computational model of the electrode. Neuron 59:379-391.

Brumberg JC, Nowak LG, McCormick DA (2000) Ionic mechanisms underlying high-frequency burst firing in supragranular cortical neurons. J Neurosci 20:4829-4843.

Brunel N, Chance FS, Fourcaud N, Abbott LF (2001) Effects of synaptic noise and filtering on the frequency response of spiking neurons. Phys Rev Lett 86:2186-2189.

Brunel N, Hakim V, Richardson MJE (2003) Firing-rate resonance in a generalized integrate-and-fire neuron with subthreshold resonance. Phys Rev E Stat Nonlin Soft Matter Phys 67:051916.

Bryant HL, Segundo JP (1976) Spike initiation by transmembrane current: a white noise analysis. J Physiol 260:279-314.

Carandini M, Mechler F, Leonard CS, Movshon JA (1996) Spike train encoding by regular-spiking cells of the visual cortex. J Neurophysiol 76:3425-3441.

Castro-Alamancos MA (2000) Origin of synchronized oscillations induced by neocortical disinhibition in vivo. J Neurosci 20:9195-9206.

Castro-Alamancos MA (2006) Vibrissa myoclonus (rhythmic retractions) driven by resonance of excitatory networks in motor cortex. J Neurophysiol 96:1691-1698.

Castro-Alamancos MA, Rigas P (2002) Synchronized oscillations caused by disinhibition in rodent neocortex are generated by recurrent synaptic activity mediated by AMPA receptors. J Physiol 542:567-581.

Castro-Alamancos MA, Rigas P, Tawara-Hirata Y (2007) Resonance $(\sim 10$
$\mathrm{Hz}$ ) of excitatory networks in motor cortex: effects of voltage-dependent ion channel blockers. J Physiol 578:173-191.

Chance FS, Abbott LF, Reyes AD (2002) Gain modulation from background synaptic input. Neuron 35:773-782.

Chen D, Fetz EE (2005) Characteristic membrane potential trajectories in primate sensorimotor cortex neurons recorded in vivo. J Neurophysiol 94:2713-2725.

Connors BW, Gutnick MJ, Prince DA (1982) Electrophysiological properties of neocortical neurons in vitro. J Neurophysiol 48:1302-1320.

Cunningham MO, Whittington MA, Bibbig A, Roopun A, LeBeau FE, Vogt A, Monyer H, Buhl EH, Traub RD (2004) A role for fast rhythmic bursting neurons in cortical gamma oscillations in vitro. Proc Natl Acad Sci U S A 101:7152-7157.

de Kock CP, Sakmann B (2008) High frequency action potential bursts $(\geq 100 \mathrm{~Hz})$ in $\mathrm{L} 2 / 3$ and L5B thick tufted neurons in anaesthetized and awake rat primary somatosensory cortex. J Physiol 586:3353-3364.

Destexhe A, Paré D (1999) Impact of network activity on the integrative properties of neocortical pyramidal neurons in vivo. J Neurophysiol 81:1531-1547.

Ellis LD, Mehaffey WH, Harvey-Girard E, Turner RW, Maler L, Dunn RJ (2007) SK channels provide a novel mechanism for the control of frequency tuning in electrosensory neurons. J Neurosci 27:9491-9502.

Fellous JM, Rudolph M, Destexhe A, Sejnowski TJ (2003) Synaptic background noise controls the input/output characteristics of single cells in an in vitro model of in vivo activity. Neuroscience 122:811-829.

Fernandez FR, White JA (2008) Artificial synaptic conductances reduce subthreshold oscillations and periodic firing in stellate cells of the entorhinal cortex. J Neurosci 28:3790-3803.

Flint AC, Connors BW (1996) Two types of network oscillations in neocortex mediated by distinct glutamate receptor subtypes and neuronal populations. J Neurophysiol 75:951-957.

Fourcaud-Trocmé N, Hansel D, van Vreeswijk C, Brunel N (2003) How spike generation mechanisms determine the neuronal response to fluctuating inputs. J Neurosci 23:11628-11640.

Fuhrmann G, Markram H, Tsodyks M (2002) Spike frequency adaptation and neocortical rhythms. J Neurophysiol 88:761-770.

Gray CM, McCormick DA (1996) Chattering cells: superficial pyramidal neurons contributing to the generation of synchronous oscillations in the visual cortex. Science 274:109-113.

Guan D, Lee JC, Tkatch T, Surmeier DJ, Armstrong WE, Foehring RC (2006) Expression and biophysical properties of Kvl channels in supragranular neocortical pyramidal neurones. J Physiol 571:371-389.

Guan D, Lee JC, Higgs MH, Spain WJ, Foehring RC (2007a) Functional roles of Kv1 channels in neocortical pyramidal neurons. J Neurophysiol 97:1931-1940.

Guan D, Tkatch T, Surmeier DJ, Armstrong WE, Foehring RC (2007b) Kv2 subunits underlie slowly inactivating potassium current in rat neocortical pyramidal neurons. J Physiol 581:941-960.

Gutfreund Y, Yarom Y, Segev I (1995) Subthreshold oscillations and resonant frequency in guinea-pig cortical neurons: physiology and modelling. J Physiol 483:621-640.

Guttman R, Feldman L, Lecar H (1974) Squid axon membrane response to white noise stimulation. Biophys J 14:941-955.

Haas JS, White JA (2002) Frequency selectivity of layer II stellate cells in the medial entorhinal cortex. J Neurophysiol 88:2422-2429.

Helmchen F, Svoboda K, Denk W, Tank DW (1999) In vivo calcium dynamics in deep-layer cortical pyramidal neurons. Nat Neurosci 2:989-996.

Higgs MH, Slee SJ, Spain WJ (2006) Diversity of gain modulation by noise in neocortical neurons: regulation by the slow afterhyperpolarization conductance. J Neurosci 26:8787-8799.

Hu H, Vervaeke K, Storm JF (2002) Two forms of electrical resonance at theta frequencies, generated by $\mathrm{M}$-current, $\mathrm{h}$-current and persistent $\mathrm{Na}^{+}$ current in rat hippocampal pyramidal cells. J Physiol 545:783-805.

Hutcheon B, Yarom Y (2000) Resonance, oscillation and the intrinsic frequency preferences of neurons. Trends Neurosci 23:216-222.

Hutcheon B, Miura RM, Puil E (1996) Subthreshold membrane resonance in neocortical neurons. J Neurophysiol 76:683-697.

Jirsch JD, Urrestarazu E, LeVan P, Olivier A, Dubeau F, Gotman J (2006) High-frequency oscillations during human focal seizures. Brain 129:1593-1608.

Köndgen H, Geisler C, Fusi S, Wang XJ, Lüscher HR, Giugliano M (2008) 
The dynamical response properties of neocortical neurons to temporally modulated noisy inputs in vitro. Cereb Cortex 18:2086-2097.

Larkum ME, Zhu JJ, Sakmann B (1999) A new cellular mechanism for coupling inputs arriving at different cortical layers. Nature 398:338-341.

Lee YW, Schetzen M (1965) Measurement of the Wiener kernels of a nonlinear system by cross-correlation. Int J Control 2:237-254.

Leung LS, Yu HW (1998) Theta-frequency resonance in hippocampal CA1 neurons in vitro demonstrated by sinusoidal current injection. J Neurophysiol 79:1592-1596.

Lisman JE (1997) Bursts as a unit of neural information: making unreliable synapses reliable. Trends Neurosci 20:38-43.

Longtin A, Doiron B, Bulsara AR (2002) Noise-induced divisive gain control in neuron models. Biosystems 67:147-156.

Lorenzon NM, Foehring RC (1992) Relationship between repetitive firing and afterhyperpolarizations in human neocortical neurons. J Neurophysiol 67:350-363.

Lukatch HS, MacIver MB (1997) Physiology, pharmacology, and topography of cholinergic neocortical oscillations in vitro. J Neurophysiol 77:2427-2445.

Lundstrom BN, Higgs MH, Spain WJ, Fairhall AL (2008) Fractional differentiation by neocortical pyramidal neurons. Nat Neurosci 11:1335-1342.

Mazzoni A, Broccard FD, Garcia-Perez E, Bonifazi P, Ruaro ME, Torre V (2007) On the dynamics of the spontaneous activity in neuronal networks. PLoS One 2:e439.

Narayanan R, Johnston D (2007) Long-term potentiation in rat hippocampal neurons is accompanied by spatially widespread changes in intrinsic oscillatory dynamics and excitability. Neuron 56:1061-1075.

Nowak LG, Azouz R, Sanchez-Vives MV, Gray CM, McCormick DA (2003) Electrophysiological classes of cat primary visual cortical neurons in vivo as revealed by quantitative analyses. J Neurophysiol 89:1541-1566.

Oswald AM, Chacron MJ, Doiron B, Bastian J, Maler L (2004) Parallel processing of sensory input by bursts and isolated spikes. J Neurosci 24:4351-4362.

Paxinos G, Watson C (1986) The rat brain in stereotaxic coordinates, Ed 2. San Diego: Academic.

Peters HC, Hu H, Pongs O, Storm JF, Isbrandt D (2005) Conditional transgenic suppression of $\mathrm{M}$ channels in mouse brain reveals functions in neuronal excitability, resonance, and behavior. Nat Neurosci 8:51-60.

Richardson MJ, Brunel N, Hakim V (2003) From subthreshold to firingrate resonance. J Neurophysiol 89:2538-2554.

Schindler KA, Goodman PH, Wieser HG, Douglas RJ (2006) Fast oscillations trigger bursts of action potentials in neocortical neurons in vitro: a quasi-white-noise analysis study. Brain Res 1110:201-210.

Schwindt PC, Spain WJ, Foehring RC, Stafstrom CE, Chubb MC, Crill WE (1988) Multiple potassium conductances and their functions in neurons from cat sensorimotor cortex in vitro. J Neurophysiol 59:424-449.

Shu Y, Hasenstaub A, Badoual M, Bal T, McCormick DA (2003) Barrages of synaptic activity control the gain and sensitivity of cortical neurons. J Neurosci 23:10388-10401.
Silva LR, Amitai Y, Connors BW (1991) Intrinsic oscillations of neocortex generated by layer 5 pyramidal neurons. Science 251:432-435.

Slee SJ, Higgs MH, Fairhall AL, Spain WJ (2005) Two-dimensional time coding in the auditory brainstem. J Neurosci 25:9978-9988.

Spain WJ, Schwindt PC, Crill WE (1991) Two transient potassium currents in layer V pyramidal neurones from cat sensorimotor cortex. J Physiol 434:591-607.

Steriade M, Timofeev I, Dürmüller N, Grenier F (1998a) Dynamic properties of corticothalamic neurons and local cortical interneurons generating fast rhythmic $(30-40 \mathrm{~Hz})$ spike bursts. J Neurophysiol 79:483-490.

Steriade M, Amzica F, Neckelmann D, Timofeev I (1998b) Spike-wave complexes and fast components of cortically generated seizures. II. Extra- and intracellular patterns. J Neurophysiol 80:1456-1479.

Teich MC, Heneghan C, Lowen SB, Ozaki T, Kaplan E (1997) Fractal character of the neural spike train in the visual system of the cat. J Opt Soc Am A Opt Image Sci Vis 14:529-546.

Thomson AM (1997) Activity-dependent properties of synaptic transmission at two classes of connections made by rat neocortical pyramidal axons in vitro. J Physiol 502:131-147.

Thomson AM, Deuchars J (1997) Synaptic interactions in neocortical local circuits: dual intracellular recordings in vitro. Cereb Cortex 7:510-522.

Thomson AM, West DC, Deuchars J (1995) Properties of single axon excitatory postsynaptic potentials elicited in spiny interneurons by action potentials in pyramidal neurons in slices of rat neocortex. Neuroscience 69:727-738.

Traub RD, Buhl EH, Gloveli T, Whittington MA (2003) Fast rhythmic bursting can be induced in layer $2 / 3$ cortical neurons by enhancing persistent $\mathrm{Na}^{+}$conductance or by blocking BK channels. J Neurophysiol 89:909-921.

van Hateren JH (1997) Processing of natural time series of intensities by the visual system of the blowfly. Vision Res 37:3407-3416.

Voss RF, Clarke J (1975) 1/fnoise in music and speech. Nature 258:317-318.

Wang XJ (1999) Fast burst firing and short-term synaptic plasticity: a model of neocortical chattering neurons. Neuroscience 89:347-362.

Watanabe J, Rozov A, Wollmuth LP (2005) Target-specific regulation of synaptic amplitudes in the neocortex. J Neurosci 25:1024-1033.

Williams SR, Atkinson SE (2007) Pathway-specific use-dependent dynamics of excitatory synaptic transmission in rat intracortical circuits. J Physiol 585:759-777.

Williams SR, Stuart GJ (1999) Mechanisms and consequences of action potential burst firing in rat neocortical pyramidal neurons. J Physiol 521:467-482.

Worrell GA, Parish L, Cranstoun SD, Jonas R, Baltuch G, Litt B (2004) High-frequency oscillations and seizure generation in neocortical epilepsy. Brain 127:1496-1506.

Yu XL, Lewis ER (1989) Studies with spike initiators: linearization by noise allows continuous signal modulation in neural networks. IEEE Trans Biomed Eng 36:36-43. 